\title{
Nesting and dressing
}

\section{Adam Rej, Matthias Staudacher and Stefan Zieme}

Max-Planck-Institut für Gravitationsphysik, Albert-Einstein-Institut, Am Mühlenberg 1, D-14476 Potsdam, Germany

E-mail: arej@aei.mpg.de, matthias@aei.mpg.de and stzieme@aei.mpg.de

Received 28 April 2007

Accepted 17 May 2007

Published 1 August 2007

Online at stacks.iop.org/JSTAT/2007/P08006

doi:10.1088/1742-5468/2007/08/P08006

Abstract. We compute the anomalous dimensions of field strength operators $\operatorname{Tr} \mathcal{F}^{L}$ in $\mathcal{N}=4$ super Yang-Mills theory (SYM) from an asymptotic nested Bethe ansatz to all-loop order. Starting from the exact solution of the one-loop problem at arbitrary $L$, we derive a single effective integral equation for the thermodynamic $L \rightarrow \infty$ limit of these dimensions. We also include the recently proposed phase factor for the $S$-matrix of the planar AdS/CFT system. The terms in the effective equation corresponding to the nesting and the dressing are structurally very similar. This hints at the physical origin of the dressing phase, which we conjecture to arise from the hidden presence of infinitely many auxiliary Bethe roots describing a non-trivial 'filled' structure of the theory's BPS vacuum. We finally show that the mechanism for creating effective nesting/dressing kernels is quite generic by also deriving the integral equation for the all-loop dimension of a certain one-loop $\mathfrak{s o}(6)$ singlet state.

Keywords: integrable quantum field theory, integrable spin chains (vertex models), quantum integrability (Bethe ansatz), solvable lattice models

ArXiv ePrint: hep-th/0702151 


\section{Contents}

1. Motivation, conclusion and overview 2

2. Excitation scheme for field strength operators 5

3. The one-loop problem $\quad 8$

3.1. Energy and higher conserved charges . . . . . . . . . . . . . . . 10

3.2. Generating polynomials . . . . . . . . . . . . . . . . 11

4. Thermodynamic limit 11

4.1. Asymptotic all-loop effective Bethe equations . . . . . . . . . . . . . 13

4.2. All-loop energy . . . . . . . . . . . . . . . . . . . . 15

5. Maximal filling and nesting 18

6. Outlook 22

Acknowledgments $\quad 23$

Appendix A. Properties of the generating polynomials 23

Appendix B. Formation of stacks $\quad 24$

B.1. Power expansion of the polynomials . . . . . . . . . . . . . 25

B.2. Emergence of the stack picture . . . . . . . . . . . . . 26

B.2.1. $n=2 L-a$ case. . . . . . . . . . . . . . 27

B.2.2. Other values of $n \ldots \ldots \ldots \ldots 27$

Appendix C. Exact results for finite $L \quad 28$

Appendix D. Emulation of the dressing phase? 29

\begin{tabular}{ll} 
References & $\mathbf{3 4}$ \\
\hline
\end{tabular}

\section{Motivation, conclusion and overview}

There is much evidence that planar $\mathcal{N}=4$ SYM theory is integrable and that its spectral problem is therefore exactly solvable. It was shown by Minahan and Zarembo that the dilatation operator in the scalar matter sector at one-loop order can be mapped to the Hamiltonian of an integrable $\mathfrak{s o}(6)$ spin chain and hence its eigenvalues can be found with a Bethe ansatz [1]. This extends to the full set of operators, leading to an integrable non-compact nearest neighbour supermagnet [2]. The special 'solvable' properties of the $\mathcal{N}=4$ model under dilatation were already hinted at by Lipatov in [3], and extend a rather generic if incomplete phenomenon in more general gauge theories such as QCD, as first shown by Belitsky, Braun, Derkachov, Korchemsky and Manashov [4].

The concept of factorized scattering, one of the hallmarks of integrability, can be extended to higher loop orders [5], and to strong coupling [6], where the gauge theory is expected to be more suitably described by a superstring theory in a curved $\operatorname{AdS}_{5} \times S^{5}$ background. Various analyses of this topic [7] led to a set of asymptotic all-loop Bethe equations for the full theory [8]. It should be stressed that quantum integrability 
remains to be proven in both gauge and string theory. In particular, on the gauge side one phenomenologically finds an integrable long-range spin chain, and the all-loop factorization of the multi-body magnon $S$-matrix into two-body processes currently has to be assumed. Correspondingly, symmetry fixes the magnon $S$-matrix only up to an overall phase factor $[5,8,9]$. The latter encodes our lack of understanding of the underlying microscopic integrable structure ${ }^{1}$. However, as was argued by Janik, the dressing phase may be constrained by invoking crossing invariance ${ }^{2}$ [11]. And indeed the string $S$ matrix satisfies crossing to the known [6,12] orders [13]. A proposal for the complete structure of the dressing phase has recently been made in [14] by combining Bethe ansatz techniques for the all-order perturbative large spin limit of Wilson twist operators [15] with conjectures by Beisert, Hernández and López [16] on the full (asymptotic) structure of the string Bethe ansatz [6].

An independent four-loop calculation strongly supports this guess [17]. This calculation scheme has been algorithmically improved and leads to agreement between field theory and the dressed Bethe ansatz with a margin of error of $0.001 \%$ [18]. Incorporating this dressing phase into the Bethe ansatz, an integral equation for the universal scaling function $f(g)$ of $\mathcal{N}=4$ gauge theory in the large spin limit was derived in [14]. Some of the analytic properties of the solution of this equation were analysed in [19], and in the leading strong coupling limit the behaviour of the scaling function as predicted by string theory [20] through the AdS/CFT correspondence agrees with the analysis of the equation [21]-[24]. The subleading order was also successfully, albeit numerically, compared in [21] to the known string result [25].

The dressing phase of [14] takes a surprisingly complex and seemingly opaque form. Arguing that it should be of a fundamental nature is, to put it mildly, unconvincing. Let us recall its structure. The phase shift $2 \theta\left(u, u^{\prime}\right)=-\mathrm{i} \log \sigma^{2}\left(u, u^{\prime}\right)$ stemming from the dressing factor $\sigma^{2}\left(u, u^{\prime}\right)$ when two magnons with rapidities $u, u^{\prime}$ pass each other (see $[8,14]$ for the notation) is

$2 \mathrm{i} \theta\left(u, u^{\prime}\right)=2 g^{2} \int_{-\infty}^{\infty} \mathrm{d} t \mathrm{e}^{\mathrm{i} t u} \mathrm{e}^{-|t| / 2} \int_{-\infty}^{\infty} \mathrm{d} t^{\prime} \mathrm{e}^{\mathrm{i} t^{\prime} u^{\prime}} \mathrm{e}^{-\left|t^{\prime}\right| / 2}\left(\hat{K}_{\mathrm{d}}\left(2 g t, 2 g t^{\prime}\right)-\hat{K}_{\mathrm{d}}\left(2 g t^{\prime}, 2 g t\right)\right)$,

where the 'magic kernel' reads

$$
\hat{K}_{\mathrm{d}}\left(t, t^{\prime}\right)=8 g^{2} \int_{0}^{\infty} \mathrm{d} t^{\prime \prime} \hat{K}_{1}\left(t, 2 g t^{\prime \prime}\right) \frac{t^{\prime \prime}}{\mathrm{e}^{t^{\prime \prime}}-1} \hat{K}_{0}\left(2 g t^{\prime \prime}, t^{\prime}\right),
$$

and the symmetric kernels $\hat{K}_{0}, \hat{K}_{1}$ are expressed with the help of Bessel functions as

$$
\begin{aligned}
& \hat{K}_{0}\left(t, t^{\prime}\right)=\frac{t J_{1}(t) J_{0}\left(t^{\prime}\right)-t^{\prime} J_{0}(t) J_{1}\left(t^{\prime}\right)}{t^{2}-t^{2}}, \\
& \hat{K}_{1}\left(t, t^{\prime}\right)=\frac{t^{\prime} J_{1}(t) J_{0}\left(t^{\prime}\right)-t J_{0}(t) J_{1}\left(t^{\prime}\right)}{t^{2}-t^{\prime 2}} .
\end{aligned}
$$

\footnotetext{
${ }^{1}$ As shown in [9], the full $S$-matrix, which is proportional to the tensor product of two copies of Shastry's $R$-matrix for the Hubbard model, satisfies the Yang-Baxter equation (see also [10]). It should be stressed, however, that Yang-Baxter symmetry of the two-body $S$-matrix is a necessary, but not sufficient condition for the integrability of a given system. An infamous counter-example is the bosonic Hubbard model.

${ }^{2}$ Crossing symmetry should also be a consequence of - rather than an axiom for-the proper microscopic formulation of the planar AdS/CFT system.
} 
This way of writing the dressing phase has a decidedly 'thermodynamic' flavour. Nevertheless, the claim of [14] is certainly that this phase should contribute to the anomalous dimensions of short operators ${ }^{3}$ at and beyond four-loop order, up until 'wrapping order'. A natural explanation of its structure could come from a hidden 'nontrivial' vacuum. Recall that this is usually the case in relativistic field theories, or else, in 'physical' ground state configurations of magnetic systems such as the Heisenberg or Hubbard antiferromagnets. What we are saying is that the dressing factor indicates that the BPS states

$$
\operatorname{Tr} \mathcal{Z}^{L}
$$

do not constitute boring 'ferromagnetic' reference vacua, but are rather states more akin to an 'antiferromagnetic', 'physical' vacuum. For reasons to be understood, BPS vacuum polarization at weak coupling appears for the first time at four-loop order. The difference, in the $\mathfrak{s u}(2)$ sector, of the AdS/CFT system to the Hubbard model [26] is then that in the latter this vacuum polarization does not appear. Toy models approximatively demonstrating a similar phenomenon in the present context have appeared previously in $[27,28]$.

In the current paper we will not rigorously analyse the underlying vacuum structure of the BPS states (1.5), which requires to go beyond the Bethe equations of [8]. In order to nevertheless prove our point, we will instead focus on another 'false vacuum', but one which may be treated with the asymptotic spectral equations in [8]. Namely, we will consider operators which at one-loop order are built from a sole field strength component $\mathcal{F}:$

$$
\operatorname{Tr} \mathcal{F}^{L}
$$

These are of interest as they are not embedded in a rank-one subsector, and are therefore described by an asymptotic nested Bethe ansatz. If $L$ is large enough, i.e. the operator is 'long', we may trust the ansatz to arbitrary loop order. In fact, we may pass to the thermodynamic limit $L \rightarrow \infty$, in which the dimension of the operators (1.6) becomes

$$
\Delta(g)=2 L+8 L g^{2} \int_{0}^{\infty} \mathrm{d} t \frac{J_{1}(2 g t)}{2 g t} \hat{\rho}(t)
$$

where $\hat{\rho}(t)$ is essentially the Fourier transform of the density of momentum-carrying firstlevel Bethe roots. As is common in thermodynamic situations, the non-linear nested Bethe equations may be turned into a system of linear integral equations for $\hat{\rho}(t)$ in conjunction with a number of further auxiliary densities describing the distribution of the higher level Bethe roots. Interestingly, the auxiliary densities may be eliminated, and one ends up with a single, effective linear equation for the principal density $\hat{\rho}(t)$. It reads

$$
\begin{aligned}
\hat{\rho}(t)=\mathrm{e}^{-2 t}(1 & \left.+\mathrm{e}^{t}\right)\left[J_{0}(2 g t)\right. \\
& -4 g^{2} t \int_{0}^{\infty} \mathrm{d} t^{\prime}\left(\frac{\mathrm{e}^{t}}{\mathrm{e}^{t}+1} \hat{K}_{0}\left(2 g t, 2 g t^{\prime}\right)+\frac{\mathrm{e}^{t^{\prime}}}{\mathrm{e}^{t^{\prime}}+1} \hat{K}_{1}\left(2 g t, 2 g t^{\prime}\right)\right) \hat{\rho}\left(t^{\prime}\right) \\
& \left.-4 g^{2} t \int_{0}^{\infty} \mathrm{d} t^{\prime}\left(\hat{K}_{\mathrm{n}}\left(2 g t, 2 g t^{\prime}\right)+\hat{K}_{\mathrm{d}}\left(2 g t, 2 g t^{\prime}\right)\right) \hat{\rho}\left(t^{\prime}\right)\right] .
\end{aligned}
$$

\footnotetext{
${ }^{3}$ Strictly speaking the phase was found in [14] by studying the large spin limit of twist operators. This is very similar to a thermodynamic limit in that it also involves infinitely many Bethe roots [15]. It would be very important to check the phase at four-loop order for a finite, short operator without taking any limit.
} 
Here $\hat{K}_{\mathrm{d}}$ is the above kernel (1.2) summarizing the effects of the dressing phase. Interestingly, together with a few further terms in the second line of (1.8), the net influence of the nesting is exerted by the kernel $\hat{K}_{\mathrm{n}}$ which reads

$$
\hat{K}_{\mathrm{n}}\left(t, t^{\prime}\right)=4 g^{2} \int_{0}^{\infty} \mathrm{d} t^{\prime \prime} \hat{K}_{1}\left(t, 2 g t^{\prime \prime}\right) \frac{t^{\prime \prime}}{\mathrm{e}^{t^{\prime \prime}}+1} \hat{K}_{0}\left(2 g t^{\prime \prime}, t^{\prime}\right) .
$$

We are confident that the reader's sharp eyes will spot the structural similarity between this nesting kernel $\hat{K}_{\mathrm{n}}$ and the dressing kernel $\hat{K}_{\mathrm{d}}$ in (1.2). In conclusion, this result suggests that the AdS/CFT phase factor [14] is generated by hidden, nested levels of a yet to be constructed final Bethe ansatz.

In the remainder of this paper we will work out the asymptotic anomalous dimensions of the operators (1.6) and derive the above equations. The detailed calculations are rather technical, and proceed as follow. In section 2 we obtain the asymptotic spectrum of these operators, which form a long-range spin chain, by applying the Bethe equations of [8]. In section 3 we solve the one-loop problem, i.e. we find the principal and auxiliary oneloop Bethe roots for the appropriate Dynkin diagram. This solution is exact for all finite values of the length $L$. We also find all one-loop higher conserved charges at arbitrary $L$. The heart of the paper is section 4 , where we work out the thermodynamic limit of our one-loop solution, find (thermodynamically) all higher loop perturbations, and reduce the resulting system of integral equations to the single effective one in (1.8). Finally, in section 5, we study a few further thermodynamic high energy states satisfying special filling conditions. Apart from exhibiting certain intriguing transcendentality properties, the examples provide further support for our picture of the origin of nesting and dressing factors. We end with a short outlook. As always when working with Bethe equations, great care has to be taken to understand the distribution of roots. The appendices contain numerous checks and considerations justifying the methodology and statements in the main body of the text.

\section{Excitation scheme for field strength operators}

We are interested in the pseudo-vacuum states $\operatorname{Tr} \mathcal{F}^{L}$ in (1.6). Here pseudo-vacuum refers to the fact that these states may serve as a reference vacuum for the one-loop Bethe ansatz [2]. In an oscillator realization of the system, see [29], $\mathcal{F}^{L}$ is the tensor product of $L$ fields $|\mathcal{F}\rangle$ where

$$
|\mathcal{F}\rangle=\mathbf{a}_{1}^{\dagger} \mathbf{a}_{1}^{\dagger}|0\rangle,
$$

and $\mathbf{a}_{1}^{\dagger}$ are bosonic creation operators. After imposing the trace condition, these states are gauge invariant, but, in contradistinction to the superficially similar BPS states $\operatorname{Tr} \mathcal{Z}^{L}$ in (1.5), not protected. In fact, their anomalous dimension is very large [29]. This type of operator was studied at one-loop order in an integrable sector of QCD in [30].

There exist alternative choices for the Dynkin diagrams of a superalgebra due to the freedom to choose each node to be either fermionic or bosonic, with the constraint that at least one node has to be fermionic. Changing from one diagram to another changes the excitation pattern of the spin chain, and sometimes even induces a change of the corresponding vacuum state. In particular, the one-loop vacuum of the standard distinguished Dynkin diagram of $\mathfrak{p s u}(2,2 \mid 4)$ is precisely given by the states $\operatorname{Tr} \mathcal{F}^{L}[2]$. Therefore no Bethe ansatz, which takes care of the diagonalization of excitations, is 


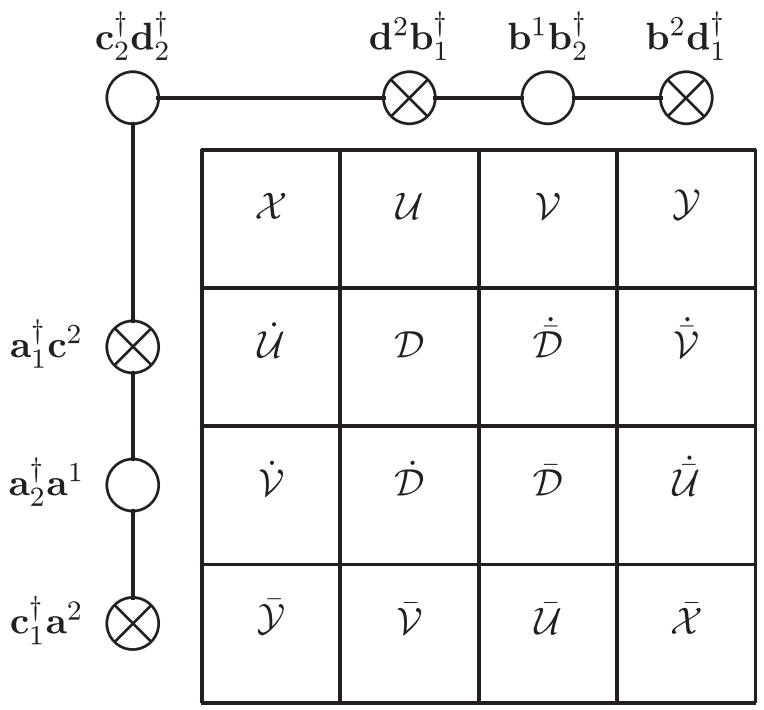

Figure 1. Oscillator realization and fundamental magnons. There are four adjoint complex scalars $\mathcal{X}, \overline{\mathcal{X}}, \mathcal{Y}, \overline{\mathcal{Y}}$, four light-cone covariant derivatives, $\mathcal{D}, \overline{\mathcal{D}}, \dot{\mathcal{D}}, \dot{\overline{\mathcal{D}}}$, and eight adjoint fermions $\mathcal{U}, \mathcal{V}, \dot{\mathcal{U}}, \dot{\mathcal{V}}, \overline{\mathcal{U}}, \overline{\mathcal{V}}, \dot{\overline{\mathcal{U}}}, \dot{\overline{\mathcal{V}}}$. The nodes of the Dynkin diagram are labelled from 1 to 7 , starting at the south-west end and going clockwise until the north-east end is reached.

necessary, and the one-loop anomalous dimension is just the vacuum energy. However, it is not known how to deform the one-loop Bethe equations of the distinguished Dynkin diagram to higher loops. The same is true for most other choices, and the long-range Bethe equations of [8] only appear to 'work' for a very specific diagram. In that diagram the vacuum is $\operatorname{Tr} \mathcal{Z}^{L}$, and $\operatorname{Tr} \mathcal{F}^{L}$ is a highly excited state with many excitations, whose momenta have to be diagonalized in order to reproduce the correct energy.

A somewhat similar pseudo-vacuum consists of a cyclic tensor product of $L$ fermions $\mathcal{U}$, i.e. $\operatorname{Tr} \mathcal{U}^{L}$, which was studied in [31]. However, while the latter lies in a rank-one subsector of the symmetry group, $\mathfrak{s u}(1 \mid 1)$, the states $\operatorname{Tr} \mathcal{F}^{L}$ are not confined to any such subsector. Correspondingly, in order to diagonalize the latter one needs a nested Bethe ansatz. The excitation pattern of Bethe roots for the higher loop Dynkin diagram of [8] reads

$\left(K_{1}, K_{2}, K_{3}, K_{4}, K_{5}, K_{6}, K_{7}\right)=(0,0,2 L-3,2 L-2, L-1, L-2, L-3)$,

where the $K_{\nu}$ is the excitation number of the $\nu$ th node of the Dynkin diagram. Clearly all but the first two nodes are highly excited.

It is interesting to understand the state $\operatorname{Tr} \mathcal{F}^{L}$ in the picture of the elementary excitations of the long-range spin chain: the $8+8$ magnons of the $\mathcal{N}=4$ model. We tabulated in figure 1 all 'fundamental magnons' in a way which allows to read of the excitation pattern in the higher loop Dynkin diagram with ease. We also included the magnon-creation operators in the oscillator picture, cf [29]. The $\mathfrak{s u}(2 \mid 2) \oplus \mathfrak{s u}(2 \mid 2)$ invariant $S$-matrix acts, respectively, on the rows and columns of figure 1. Exciting the central fourth node $K_{4}$ times inserts $K_{4} \mathcal{X}$ bosons into the BPS vacuum $\mathcal{Z}^{L}$. The $K_{4}$ Bethe roots of the central node parametrize the momenta of these magnons. Next, exciting $K_{5}$ times the fifth node converts $K_{5}$ of the $\mathcal{X}$ bosons into $\mathcal{U}$ fermions. The process continues until 
all magnons contained in a given state are created. It follows from $(2.2)$ that the fields $\mathcal{F}$ in $\operatorname{Tr} \mathcal{F}^{L}$ are not elementary. Instead, each $\mathcal{F}$ is essentially equivalent to a composite of two fermions $\dot{\mathcal{U}}$ and $\dot{\overline{\mathcal{V}}}$ minus one field $\mathcal{Z}$. Let us explain this in more detail for a single $\mathcal{F}$. The two fermions and the background field $\mathcal{Z}$ are expressed in terms of the oscillators as

$$
\dot{\mathcal{U}}=\mathbf{a}_{1}^{\dagger} \mathbf{c}_{4}^{\dagger}|0\rangle \quad \dot{\overline{\mathcal{V}}}=\mathbf{a}_{1}^{\dagger} \mathbf{c}_{3}^{\dagger}|0\rangle \quad \mathcal{Z}=\mathbf{c}_{3}^{\dagger} \mathbf{c}_{4}^{\dagger}|0\rangle
$$

and form a $\mathcal{F}$, cf $(2.1)$, while inserting another $\mathcal{Z}$ :

$$
\dot{\mathcal{U}} \dot{\overline{\mathcal{V}}}=\mathbf{a}_{1,1}^{\dagger} \mathbf{c}_{4,1}^{\dagger} \mathbf{a}_{1,2}^{\dagger} \mathbf{c}_{3,2}^{\dagger}|0\rangle_{1} \otimes|0\rangle_{2} \leftrightarrow \mathcal{F} \mathcal{Z}=\mathbf{a}_{1,1}^{\dagger} \mathbf{a}_{1,1}^{\dagger} \mathbf{c}_{3,2}^{\dagger} \mathbf{c}_{4,2}^{\dagger}|0\rangle_{1} \otimes|0\rangle_{2} .
$$

We see that each $\mathcal{F}$ corresponds to two magnon excitations $\dot{\mathcal{U}} \overline{\mathcal{V}}$. Removing $L$ background fields $\mathcal{Z}$ does not change the excitation number, but reduces the length (as well as the $R$-charge) by $L$ units. Correspondingly, the state $\operatorname{Tr} \mathcal{F}^{L}$ has, at one-loop order, $2 L$ excitations living on a lattice of $L$ sites. Beyond one-loop order, this length is not conserved.

In order to write down the Bethe equations it is useful to define spectral parameters $x^{ \pm}, u^{ \pm}, u$ which are interrelated by

$$
x(u)=\frac{u}{2}\left(1+\sqrt{1-4 \frac{g^{2}}{u^{2}}}\right), \quad x^{ \pm}=x\left(u^{ \pm}\right), \quad u^{ \pm}=u \pm \frac{\mathrm{i}}{2},
$$

and the coupling constant $g$ is proportional to the square root of the 't Hooft coupling constant

$$
g=\frac{\sqrt{\lambda}}{4 \pi}
$$

These parameters are assigned double indices, i.e. the Bethe equations are written in terms of $x_{\nu, k}^{ \pm}, u_{\nu, k}$ where $\nu=1, \ldots, 7$ denotes the nesting level (or node number of the Dynkin diagram), and $k=1, \ldots, K_{\nu}$ labels the Bethe roots of level $\nu$.

The Bethe equations for the specific excitation pattern (2.2) are given by (cf [8])

$$
\begin{aligned}
& 1= \prod_{j=1}^{2 L-2} \frac{x_{3, k}-x_{4, j}^{+}}{x_{3, k}-x_{4, j}^{-}} \\
&\left(\frac{x_{4, k}^{+}}{x_{4, k}^{-}}\right)^{L}=\prod_{\substack{j=1 \\
j \neq k}}^{2 L-2} \frac{x_{4, k}^{+}-x_{4, j}^{-}}{x_{4, k}^{-}-x_{4, j}^{+}} \frac{1-g^{2} / x_{4, k}^{+} x_{4, j}^{-}}{1-g^{2} / x_{4, k}^{-} x_{4, j}^{+}} \sigma^{2}\left(u_{4, k}, u_{4, j}\right) \\
& \quad \times \prod_{j=1}^{2 L-3} \frac{x_{4, k}^{-}-x_{3, j}}{x_{4, k}^{+}-x_{3, j}} \prod_{j=1}^{L-1} \frac{x_{4, k}^{-}-x_{5, j}}{x_{4, k}^{+}-x_{5, j}} \prod_{j=1}^{L-3} \frac{1-g^{2} / x_{4, k}^{-} x_{7, j}}{1-g^{2} / x_{4, k}^{+} x_{7, j}} \\
& 1=\prod_{j=1}^{L-2} \frac{u_{5, k}-u_{6, j}+\mathrm{i} / 2}{u_{5, k}-u_{6, j}-\mathrm{i} / 2} \prod_{j=1}^{2 L-2} \frac{x_{5, k}-x_{4, j}^{+}}{x_{5, k}-x_{4, j}^{-}} \\
& \quad \prod_{j=1}^{L-2} \frac{u_{6, k}-u_{6, j}-\mathrm{i}}{u_{6, k}-u_{6, j}+\mathrm{i}} \prod_{j=1}^{L-1} \frac{u_{6, k}-u_{5, j}+\mathrm{i} / 2}{u_{6, k}-u_{5, j}-\mathrm{i} / 2} \prod_{j=1}^{L-3} \frac{u_{6, k}-u_{7, j}+\mathrm{i} / 2}{u_{6, k}-u_{7, j}-\mathrm{i} / 2} \\
& 1=\prod_{j=1}^{L-2} \frac{u_{7, k}-u_{6, j}+\mathrm{i} / 2}{u_{7, k}-u_{6, j}-\mathrm{i} / 2} \prod_{j=1}^{2 L-2} \frac{1-g^{2} / x_{7, k} x_{4, j}^{+}}{1-g^{2} / x_{7, k} x_{4, j}^{-}},
\end{aligned}
$$

where the dressing factor $\sigma^{2}\left(u_{k}, u_{j}\right)=\mathrm{e}^{2 \mathrm{i} \theta\left(u_{k}, u_{j}\right)}$ is given by (1.1). 
The (asymptotic) anomalous dimensions of an operator can then be deduced from the energy $E(g)$ of the long-range spin chain by

$$
\Delta=\Delta_{0}+2 g^{2} E(g),
$$

where $\Delta_{0}$ denotes the operator's classical dimension. In the case of the field strength operators (1.6) it is given by $\Delta_{0}=2 L$. In turn, this energy is found from the 'momentumcarrying' Bethe roots living on the central (i.e. fourth) node as

$$
E(g)=\sum_{k=1}^{2 L-2}\left(\frac{\mathrm{i}}{x_{4, k}^{+}}-\frac{\mathrm{i}}{x_{4, k}^{-}}\right)
$$

Let us note that the state $\dot{\mathcal{F}}^{L}$, which is a tensor product of

$$
|\dot{\mathcal{F}}\rangle=\mathbf{b}_{1}^{\dagger} \mathbf{b}_{1}^{\dagger} \mathbf{c}_{1}^{\dagger} \mathbf{c}_{2}^{\dagger} \mathbf{c}_{3}^{\dagger} \mathbf{c}_{4}^{\dagger}|0\rangle
$$

has mirror-inverted excitation numbers and hence the same Bethe equations (2.7).

We will firstly solve the one-loop problem at finite $L$, and secondly construct the thermodynamic limit $L \rightarrow \infty$. Subsequently we will focus on the all-loop equations and derive an integral equation for the corresponding root density.

\section{The one-loop problem}

The one-loop Bethe equations for the field strength operators $\operatorname{Tr} \mathcal{F}^{L}$ are obtained from the asymptotic all-loop equations $(2.7)$ by taking the limit $g \rightarrow 0$. This yields

$$
\begin{aligned}
& 1=\prod_{j=1}^{2 L-2} \frac{u_{3, k}-u_{4, j}-\mathrm{i} / 2}{u_{3, k}-u_{4, j}+\mathrm{i} / 2} \\
& \left(\frac{u_{4, k}+\mathrm{i} / 2}{u_{4, k}-\mathrm{i} / 2}\right)^{L}=\prod_{\substack{j=1 \\
j \neq k}}^{2 L-2} \frac{u_{4, k}-u_{4, j}+\mathrm{i}}{u_{4, k}-u_{4, j}-\mathrm{i}} \prod_{j=1}^{2 L-3} \frac{u_{4, k}-u_{3, j}-\mathrm{i} / 2}{u_{4, k}-u_{3, j}+\mathrm{i} / 2} \prod_{j=1}^{L-1} \frac{u_{4, k}-u_{5, j}-\mathrm{i} / 2}{u_{4, k}-u_{5, j}+\mathrm{i} / 2} \\
& 1=\prod_{j=1}^{L-2} \frac{u_{5, k}-u_{6, j}+\mathrm{i} / 2}{u_{5, k}-u_{6, j}-\mathrm{i} / 2} \prod_{j=1}^{2 L-2} \frac{u_{5, k}-u_{4, j}-\mathrm{i} / 2}{u_{5, k}-u_{4, j}+\mathrm{i} / 2} \\
& 1=\prod_{\substack{j=1 \\
j \neq k}}^{L-2} \frac{u_{6, k}-u_{6, j}-\mathrm{i}}{u_{6, k}-u_{6, j}+\mathrm{i}} \prod_{j=1}^{L-1} \frac{u_{6, k}-u_{5, j}+\mathrm{i} / 2}{u_{6, k}-u_{5, j}-\mathrm{i} / 2} \prod_{j=1}^{L-3} \frac{u_{6, k}-u_{7, j}+\mathrm{i} / 2}{u_{6, k}-u_{7, j}-\mathrm{i} / 2} \\
& 1=\prod_{j=1}^{L-2} \frac{u_{7, k}-u_{6, j}+\mathrm{i} / 2}{u_{7, k}-u_{6, j}-\mathrm{i} / 2} .
\end{aligned}
$$


These can be further simplified with a suitable dualization, see e.g. [32] and references therein. We start by introducing the polynomial $P(u)$ for the $u_{3}$ roots as

$P(u)=\prod_{j=1}^{2 L-2}\left(u-u_{4, j}+\frac{\mathrm{i}}{2}\right)-\prod_{j=1}^{2 L-2}\left(u-u_{4, j}-\frac{\mathrm{i}}{2}\right)=\mathrm{i}(2 L-2) \prod_{j=1}^{2 L-3}\left(u-u_{3, j}\right)$.

It is straightforward to find

$$
\frac{P\left(u_{4, k}+\mathrm{i} / 2\right)}{P\left(u_{4, k}-\mathrm{i} / 2\right)}=\prod_{\substack{j=1 \\ j \neq k}}^{2 L-2} \frac{u_{4, k}-u_{4, j}+\mathrm{i}}{u_{4, k}-u_{4, j}-\mathrm{i}}=\prod_{j=1}^{2 L-3} \frac{u_{4, k}-u_{3, j}+\mathrm{i} / 2}{u_{4, k}-u_{3, j}-\mathrm{i} / 2} .
$$

Applying the same procedure to the roots $u_{7}$ and $u_{6}$ we reach an effective system of equations, namely

$$
\begin{aligned}
& \left(\frac{u_{4, k}+\mathrm{i} / 2}{u_{4, k}-\mathrm{i} / 2}\right)^{L}=\prod_{j=1}^{L-1} \frac{u_{4, k}-u_{5, j}-\mathrm{i} / 2}{u_{4, k}-u_{5, j}+\mathrm{i} / 2} \\
& 1=\prod_{\substack{j=1 \\
j \neq k}}^{L-1} \frac{u_{5, k}-u_{5, j}+\mathrm{i}}{u_{5, k}-u_{5, j}-\mathrm{i}} \prod_{j=1}^{2 L-2} \frac{u_{5, k}-u_{4, j}-\mathrm{i} / 2}{u_{5, k}-u_{4, j}+\mathrm{i} / 2} .
\end{aligned}
$$

By introducing the polynomial $Q_{4}(u)$ for the momentum-carrying roots $u_{4}$ as

$$
\begin{aligned}
Q_{4}(u) & =\left(u+\frac{\mathrm{i}}{2}\right) \prod_{j=1}^{L-1}\left(u-u_{5, j}+\frac{\mathrm{i}}{2}\right)-\left(u-\frac{\mathrm{i}}{2}\right) \prod_{j=1}^{L-1}\left(u-u_{5, j}-\frac{\mathrm{i}}{2}\right) \\
& =\mathrm{i}(2 L-1) \prod_{j=1}^{2 L-2}\left(u-u_{4, j}\right)
\end{aligned}
$$

and noting that

$$
\frac{Q_{4}\left(u_{5, k}+\mathrm{i} / 2\right)}{Q_{4}\left(u_{5, k}-\mathrm{i} / 2\right)}=\left(\frac{u_{5, k}+\mathrm{i}}{u_{5, k}-\mathrm{i}}\right)^{L} \prod_{\substack{j=1 \\ j \neq k}}^{L-1} \frac{u_{5, k}-u_{5, j}+\mathrm{i}}{u_{5, k}-u_{5, j}-\mathrm{i}}=\prod_{j=1}^{2 L-2} \frac{u_{5, k}-u_{4, j}+\mathrm{i} / 2}{u_{5, k}-u_{4, j}-\mathrm{i} / 2},
$$

we find that (3.3) simplifies enormously to the free equation

$$
\left(\frac{u_{5, k}+\mathrm{i}}{u_{5, k}-\mathrm{i}}\right)^{L}=1 \text {. }
$$

This is solved by

$$
u_{5, k}=\cot \left(\frac{\pi k}{L}\right), \quad k=1, \ldots, L-1 .
$$

Plugging this solution back into equation (3.2) we infer that $u_{4, k}$ are roots of the equation

$$
\left(\frac{u+\mathrm{i} / 2}{u-\mathrm{i} / 2}\right)^{L}=\prod_{j=1}^{L-1} \frac{u-\cot (\pi j / L)-\mathrm{i} / 2}{u-\cot (\pi j / L)+\mathrm{i} / 2} .
$$


On the other hand, the $u_{5}$ roots are zeros of the polynomial $Q_{5}(u)$

$$
Q_{5}(u)=(u+\mathrm{i})^{L}-(u-\mathrm{i})^{L},
$$

such that we can rewrite (3.4) as

$$
Q_{4}(u)=\left(u+\frac{\mathrm{i}}{2}\right)^{L} Q_{5}\left(u+\frac{\mathrm{i}}{2}\right)-\left(u-\frac{\mathrm{i}}{2}\right)^{L} Q_{5}\left(u-\frac{\mathrm{i}}{2}\right) .
$$

We have therewith derived a polynomial $Q_{4}(u)$ of degree $2 L-2$

$$
\begin{aligned}
Q_{4}(u)=(u+ & \left.\frac{\mathrm{i}}{2}\right)^{L}\left(\left(u+\frac{3}{2} \mathrm{i}\right)^{L}-\left(u-\frac{1}{2} \mathrm{i}\right)^{L}\right) \\
& +\left(u-\frac{\mathrm{i}}{2}\right)^{L}\left(\left(u-\frac{3}{2} \mathrm{i}\right)^{L}-\left(u+\frac{1}{2} \mathrm{i}\right)^{L}\right),
\end{aligned}
$$

whose zeros correspond to the exact one-loop roots $u_{4}$ for arbitrary, finite L. All roots of this polynomial are real (for a proof, see appendix A).

\subsection{Energy and higher conserved charges}

Since the $u_{4, k}$ are the roots of (3.9) we can write

$$
Q_{4}(u)=-2 L(2 L-1) \prod_{j=1}^{2 L-2}\left(u-u_{4, j}\right)
$$

This allows us to express the energy $E_{\mathcal{F} L}(g)=E_{\mathcal{F}^{L}}+\mathcal{O}\left(g^{2}\right)$ (2.9) in the one-loop approximation $E_{\mathcal{F} L}$ in terms of $Q_{4}(u)$

$$
E_{\mathcal{F} L}=\sum_{j=1}^{2 L-2} \frac{1}{1 / 4+u_{4, j}^{2}}=\mathrm{i}\left(\frac{Q_{4}^{\prime}(\mathrm{i} / 2)}{Q_{4}(\mathrm{i} / 2)}-\frac{Q_{4}^{\prime}(-\mathrm{i} / 2)}{Q_{4}(-\mathrm{i} / 2)}\right) .
$$

On the other hand, using the explicit form (3.9) we derive

$$
\frac{Q_{4}^{\prime}(\mathrm{i} / 2)}{Q_{4}(\mathrm{i} / 2)}=-\frac{3}{2} \mathrm{i} L, \quad \frac{Q_{4}^{\prime}(-\mathrm{i} / 2)}{Q_{4}(-\mathrm{i} / 2)}=\frac{3}{2} \mathrm{i} L,
$$

and thus verify that the anomalous dimensions (2.8) of the operators $\operatorname{Tr} \mathcal{F}^{L}$ are indeed, to one-loop order $[29,2]$,

$$
\Delta_{\mathcal{F} L}=2 L+6 L g^{2}+\mathcal{O}\left(g^{4}\right) .
$$

Incidentally, it is straightforward to generalize the above method to all higher conserved spin chain charges, by noting that

$$
\begin{aligned}
q_{r} & =\frac{\mathrm{i}}{(r-1)(r-2) !}\left(\left.\frac{\mathrm{d}^{r-1}}{\mathrm{~d} u^{r-1}} \log Q_{4}(u)\right|_{u=+\mathrm{i} / 2}-\left.\frac{\mathrm{d}^{r-1}}{\mathrm{~d} u^{r-1}} \log Q_{4}(u)\right|_{u=-\mathrm{i} / 2}\right) \\
& =L \frac{\mathrm{i}}{r-1}\left(1+\frac{1}{2^{r-1}}\right)\left(\mathrm{i}^{r+1}-(-\mathrm{i})^{r+1}\right) .
\end{aligned}
$$

It is clear that all odd charges vanish. Note that the $c_{r}$ in (3.20) of [32] for the 'Beast' diagram are given by $q_{r} / L$. 


\subsection{Generating polynomials}

Starting from the set of all-loop Bethe ansatz equations (2.7) and dualizing the $u_{3}$ and $u_{7}$ roots [8] we obtain

$$
\begin{aligned}
& \left(\frac{x_{4, k}^{+}}{x_{4, k}^{-}}\right)^{L}=\prod_{\substack{j=1 \\
j \neq k}}^{2 L-2} \frac{x_{4, k}^{-}-x_{4, j}^{+}}{x_{4, k}^{+}-x_{4, j}^{-}} \frac{1-\frac{g^{2}}{x_{4, k}^{+} x_{4, j}^{-}}}{1-\frac{g^{2}}{x_{4, k}^{-} x_{4, j}^{+}}} \sigma^{2}\left(u_{4, k}, u_{4, j}\right) \prod_{j=1}^{2 L-4} \frac{x_{4, k}^{+}-\tilde{x}_{5, j}}{x_{4, k}^{-}-\tilde{x}_{5, j}} \\
& 1=\prod_{j=1}^{L-2} \frac{\tilde{u}_{5, k}-u_{6, j}+\mathrm{i} / 2}{\tilde{u}_{5, k}-u_{6, j}-\mathrm{i} / 2} \prod_{j=1}^{2 L-2} \frac{\tilde{x}_{5, k}-x_{4, j}^{+}}{\tilde{x}_{5, k}-x_{4, j}^{-}} \\
& 1=\prod_{\substack{j=1 \\
j \neq k}}^{L-2} \frac{u_{6, k}-u_{6, j}+\mathrm{i}}{u_{6, k}-u_{6, j}-\mathrm{i}} \prod_{j=1}^{2 L-4} \frac{u_{6, k}-\tilde{u}_{5, j}-\mathrm{i} / 2}{u_{6, k}-\tilde{u}_{5, j}+\mathrm{i} / 2} .
\end{aligned}
$$

Here, $\tilde{u}_{5}$ are the $2 L-4$ roots dual to $u_{5}$. Note that they are not given by (3.7) anymore. It is however an easy task to derive the polynomials which generate the one-loop roots for this set of equations. One of them, generating the $u_{4}$ roots, is already known, see (3.9). The polynomials generating the $\tilde{u}_{5}$ and the $u_{6}$ roots are given, respectively, by

$$
\begin{aligned}
Q_{5}(v)=3 v^{2 L} & +(-\mathrm{i}+v)^{L}(-2 \mathrm{i}+v)^{L}+(2 \mathrm{i}+v)^{L}\left((\mathrm{i}+v)^{L}+(-2 \mathrm{i}+v)^{L}\right) \\
& -v^{L}\left((-\mathrm{i}+v)^{L}+(\mathrm{i}+v)^{L}+2(-2 \mathrm{i}+v)^{L}+2(2 \mathrm{i}+v)^{L}\right)
\end{aligned}
$$

and

$Q_{6}(w)=\left(w+\frac{3}{2} \mathrm{i}\right)^{L}+\left(w-\frac{3}{2} \mathrm{i}\right)^{L}-\left(w+\frac{1}{2} \mathrm{i}\right)^{L}-\left(w-\frac{1}{2} \mathrm{i}\right)^{L}$.

Some properties of these polynomials are studied in appendix A.

\section{Thermodynamic limit}

In this section we will demonstrate how to construct the thermodynamic limit and how to find the root densities using the generating polynomials defined in (3.9), (3.15), (3.16). We will present the method, taking $Q_{6}(w)$ in (3.16) as an example.

Let us define the density of roots as

$$
\rho_{L, 6}(z)=\frac{1}{L} \sum_{i=1}^{L-2} \delta\left(z-w_{i}\right)
$$

Using this definition we can write (3.16) as

$$
Q_{6}(w)=\prod_{i=1}^{L-2}\left(w-w_{i}\right)=\exp \left(L \int_{-\infty}^{\infty} \rho_{L, 6}(z) \log (w-z) \mathrm{d} z\right)
$$

and easily obtain an integral equation for the density

$$
\int_{-\infty}^{\infty} \frac{\rho_{L, 6}(z)}{w-z} \mathrm{~d} z=\frac{1}{L} \frac{Q_{6, L}(w)^{\prime}}{Q_{6, L}(w)}=V_{L}(w)
$$


Taking the limit $L \rightarrow \infty$ and using the identity

$$
\frac{1}{x+\mathrm{i} \epsilon}-\frac{1}{x-\mathrm{i} \epsilon}=-2 \pi \mathrm{i} \delta(x)
$$

we derive the formula

$$
\rho_{6}(w)=\frac{\mathrm{i}}{2 \pi}(V(w+\mathrm{i} \epsilon)-V(w-\mathrm{i} \epsilon)),
$$

where

$$
\rho_{6}(u)=\lim _{L \rightarrow \infty} \rho_{L, 6}(u) \quad \text { and } \quad V(w)=\lim _{L \rightarrow \infty} V_{L}(w) .
$$

It follows from the above formula that $V(w)$ is necessarily discontinuous across the real axis (i.e. it has an infinite cut there). To find $V(w)$ we compute

$$
\begin{aligned}
V_{L}(w)=\frac{1}{L} & \frac{Q_{6, L}^{\prime}(w)}{Q_{6, L}(w)} \\
& =\frac{(w+(3 / 2) \mathrm{i})^{L-1}+(w-(3 / 2) \mathrm{i})^{L-1}-(w+(1 / 2) \mathrm{i})^{L-1}-(w-(1 / 2) \mathrm{i})^{L-1}}{(w+(3 / 2) \mathrm{i})^{L}+(w-(3 / 2) \mathrm{i})^{L}-(w+(1 / 2) \mathrm{i})^{L}-(w-(1 / 2) \mathrm{i})^{L}} .
\end{aligned}
$$

Taking the limit is straightforward

$$
V(w) \equiv \lim _{L \rightarrow \infty} V_{L}(w)= \begin{cases}\frac{1}{w+(3 / 2) \mathrm{i}}, & \operatorname{Im}(w)>0 \\ \frac{1}{w-(3 / 2) \mathrm{i}}, & \operatorname{Im}(w)<0 .\end{cases}
$$

Using (4.5) we find for the density

$$
\rho_{6}(w) \equiv \lim _{L \rightarrow \infty} \rho_{L, 6}(w)=\frac{1}{2 \pi} \frac{3}{w^{2}+9 / 4} .
$$

The same procedure can be used to find the density for the momentum-carrying roots

$$
\rho_{4}(u)=\frac{1}{2 \pi}\left(\frac{1}{u^{2}+1 / 4}+\frac{3}{u^{2}+9 / 4}\right),
$$

which is a continuum limit of

$$
\rho_{L, 4}(z)=\frac{1}{L} \sum_{i=1}^{2 L-2} \delta\left(z-u_{i}\right) .
$$

As a validity check we can show that

$$
\begin{aligned}
q_{r} & =L \frac{\mathrm{i}}{r-1} \int_{-\infty}^{\infty} \rho_{4}(u)\left(\frac{1}{(u+\mathrm{i} / 2)^{r-1}}-\frac{1}{(u-\mathrm{i} / 2)^{r-1}}\right) \\
& =L \frac{\mathrm{i}}{r-1}\left(1+\frac{1}{2^{r-1}}\right)\left(\mathrm{i}^{r+1}-(-\mathrm{i})^{r+1}\right),
\end{aligned}
$$

which is in agreement with the earlier result (3.11). 
Let us now direct our attention to the density of roots of flavour $u_{5}$. From the analysis of the generating polynomial we infer that these roots form two strings along $\pm \mathrm{i} / 2$ (see appendix $\mathrm{B}$ for details) on the complex plane and we have to integrate over two contours

$$
\int_{-\infty+\mathrm{i} / 2}^{\infty+\mathrm{i} / 2} \frac{\rho_{5}\left(v^{+}\right)}{u-v^{+}} \mathrm{d} v^{+}+\int_{-\infty-\mathrm{i} / 2}^{\infty-\mathrm{i} / 2} \frac{\rho_{5}\left(v^{-}\right)}{u-v^{-}} \mathrm{d} v^{-}=\Lambda(u),
$$

where $\Lambda(u)$ is given by

$$
\Lambda(u)=\lim _{L \rightarrow \infty} \frac{1}{L} \partial_{u} \log Q_{5, L}(u)= \begin{cases}\frac{1}{u+\mathrm{i}}+\frac{1}{u+2 \mathrm{i}}, & \operatorname{Im}(u)>\frac{1}{2} \\ \frac{1}{u-2 \mathrm{i}}+\frac{1}{u+2 \mathrm{i}}, & -\frac{1}{2}<\operatorname{Im}(u)<\frac{1}{2} \\ \frac{1}{u-\mathrm{i}}+\frac{1}{u-2 \mathrm{i}}, & \operatorname{Im}(u)<-\frac{1}{2} .\end{cases}
$$

The solution of (4.13) is

$$
\rho_{5}\left(v \pm \frac{\mathrm{i}}{2}\right)=\rho_{6}(v)
$$

Hence, two roots $u_{5}^{ \pm}$form a stack with one root of type $u_{6}$ in the centre such that $u_{5}^{ \pm}=\left(u_{6} \pm \mathrm{i} / 2\right)$. The concept of stacks was introduced and studied in the context of the nested Bethe ansatz of 'ferromagnetic' root distributions [32]. Here we find the same stack picture in the context of a long operator akin to an 'antiferromagnetic' state. The emergence of the stack picture for our case is rigorously established in appendix B.

\subsection{Asymptotic all-loop effective Bethe equations}

In the thermodynamic limit only the centres of the stacks receive quantum corrections, i.e. $u_{5, k}(g) \approx u_{6, k}(g)+\mathrm{i} / 2$ and $u_{5, k+L-2}(g) \approx u_{6, k}(g)-\mathrm{i} / 2$ for $k=1, \ldots, L-2$. The effective set of Bethe equations then reads ${ }^{4}$

$$
\begin{aligned}
& \left(\frac{x_{4, k}^{+}}{x_{4, k}^{-}}\right)^{L} \approx \prod_{\substack{j=1 \\
j \neq k}}^{2 L-2} \frac{x_{4, k}^{-}-x_{4, j}^{+}}{x_{4, k}^{+}-x_{4, j}^{-}} \frac{1-g^{2} / x_{4, k}^{+} x_{4, j}^{-}}{1-g^{2} / x_{4, k}^{-} x_{4, j}^{+}} \sigma^{2}\left(u_{4, k}, u_{4, j}\right) \prod_{j=1}^{L-2} \frac{x_{4, k}^{+}-x_{6, j}^{-}}{x_{4, k}^{-}-x_{6, j}^{+}} \frac{1-g^{2} / x_{4, k}^{-} x_{6, j}^{-}}{1-g^{2} / x_{4, k}^{+} x_{6, j}^{+}} \\
& 1 \approx \prod_{\substack{j=1 \\
j \neq k}}^{L-2} \frac{u_{6, k}-u_{6, j}+\mathrm{i}}{u_{6, k}-u_{6, j}-\mathrm{i}} \prod_{j=1}^{2 L-2} \frac{x_{6, k}^{-}-x_{4, j}^{+}}{x_{6, k}^{+}-x_{4, j}^{-}} \frac{1-g^{2} / x_{6, k}^{-} x_{4, j}^{-}}{1-g^{2} / x_{6, k}^{+} x_{4, j}^{+}}
\end{aligned}
$$

where we have used the identity

$$
\left(x_{k}^{ \pm}-x_{j}^{ \pm}\right)=\frac{u_{k}-u_{j}}{1-g^{2} / x_{k}^{ \pm} x_{j}^{ \pm}} .
$$

Note that (3.13) splits up into two equations with $k$ running from $k=1, \ldots, L-2$ and $k=L-2+1, \ldots, 2 L-2$ respectively, which can by multiplied with each other to

${ }^{4}$ The use of ' $\approx$ ' instead of ' $=$ ' indicates that these equations are strictly speaking not exactly valid for finite $L$ due to differences of $\mathcal{O}(1 / L)$ for the generating polynomials. See appendix B for more details. 
get (4.17), while equation (3.14) is identically satisfied. These effective equations describe the scattering of the roots $u_{4}$ with the centres of the stacks. Rewriting (4.16) and (4.17) as

$$
\begin{gathered}
\left(\frac{x_{4, k}^{+}}{x_{4, k}^{-}}\right)^{L} \approx \prod_{\substack{j=1 \\
j \neq k}}^{2 L-2} \frac{u_{4, k}-u_{4, j}-\mathrm{i}}{u_{4, k}-u_{4, j}+\mathrm{i}}\left(\frac{1-g^{2} / x_{4, k}^{+} x_{4, j}^{-}}{1-g^{2} / x_{4, k}^{-} x_{4, j}^{+}}\right)^{2} \sigma^{2}\left(u_{4, k}, u_{4, j}\right) \\
\times \prod_{j=1}^{L-2} \frac{u_{4, k}-u_{6, j}+\mathrm{i}}{u_{4, k}-u_{6, j}-\mathrm{i}} \frac{1-g^{2} / x_{4, k}^{-} x_{6, j}^{+}}{1-g^{2} / x_{4, k}^{+} x_{6, j}^{-}} \frac{1-g^{2} / x_{4, k}^{-} x_{6, j}^{-}}{1-g^{2} / x_{4, k}^{+} x_{6, j}^{+}} \\
1 \approx \prod_{\substack{j=1 \\
j \neq k}}^{L-2} \frac{u_{6, k}-u_{6, j}+\mathrm{i}}{u_{6, k}-u_{6, j}-\mathrm{i}} \prod_{j=1}^{2 L-2} \frac{u_{6, k}-u_{4, j}-\mathrm{i}}{u_{6, k}-u_{4, j}+\mathrm{i}} \frac{1-g^{2} / x_{6, k}^{+} x_{4, j}^{-}}{1-g^{2} / x_{6, k}^{-} x_{4, j}^{+}} \frac{1-g^{2} / x_{6, k}^{-} x_{4, j}^{-}}{1-g^{2} / x_{6, k}^{+} x_{4, j}^{+}}
\end{gathered}
$$

and taking the logarithm of these equations leads to

$$
\begin{aligned}
\frac{1}{\mathrm{i}}\left(\log x_{4, k}^{+}-\right. & \left.\log x_{4, k}^{-}\right) \\
\approx & 2 \pi \frac{n_{k}}{L}+\frac{1}{\mathrm{i} L} \sum_{\substack{j=1 \\
j \neq k}}^{2 L-2} \log \frac{u_{4, k}-u_{4, j}-\mathrm{i}}{u_{4, k}-u_{4, j}+\mathrm{i}} \\
& +\frac{1}{\mathrm{i} L} \sum_{\substack{j=1 \\
j \neq k}}^{2 L-2}\left(2 \log \frac{1-g^{2} / x_{4, k}^{+} x_{4, j}^{-}}{1-g^{2} / x_{4, k}^{-} x_{4, j}^{+}}+2 \mathrm{i} \theta\left(u_{4, k}, u_{4, j}\right)\right) \\
& +\frac{1}{\mathrm{i} L} \sum_{j=1}^{L-2}\left(\log \frac{u_{4, k}-u_{6, j}+\mathrm{i}}{u_{4, k}-u_{6, j}-\mathrm{i}}+\log \frac{1-g^{2} / x_{4, k}^{-} x_{6, j}^{+}}{1-g^{2} / x_{4, k}^{+} x_{6, j}^{-}}+\log \frac{1-g^{2} / x_{4, k}^{-} x_{6, j}^{-}}{1-g^{2} / x_{4, k}^{+} x_{6, j}^{+}}\right)
\end{aligned}
$$

where the dressing phase shift $2 \mathrm{i} \theta$ is given in (1.1), and to

$$
\begin{aligned}
0 \approx 2 \pi \frac{m_{k}}{L}+ & \frac{1}{\mathrm{i} L} \sum_{\substack{j=1 \\
j \neq k}}^{L-2} \log \frac{u_{6, k}-u_{6, j}+\mathrm{i}}{u_{6, k}-u_{6, j}-\mathrm{i}} \\
& +\frac{1}{\mathrm{i} L} \sum_{j=1}^{2 L-2}\left(\log \frac{u_{6, k}-u_{4, j}-\mathrm{i}}{u_{6, k}-u_{4, j}+\mathrm{i}}+\log \frac{1-g^{2} / x_{6, k}^{+} x_{4, j}^{-}}{1-g^{2} / x_{6, k}^{-} x_{4, j}^{+}}+\log \frac{1-g^{2} / x_{6, k}^{-} x_{4, j}^{-}}{1-g^{2} / x_{6, k}^{+} x_{4, j}^{+}}\right) .
\end{aligned}
$$

The ambiguity in the choice of branch of the logarithm is encoded in the mode numbers $n_{k}$ and $m_{k}$, for which we introduce mode functions $n_{k}=n\left(u_{4, k}\right)$ and $m_{k}=m\left(u_{6, k}\right)$.

As usual we will now replace the sums by integrals as $L \rightarrow \infty$ and introduce new variables $\xi=n\left(u_{4}\right) / L$ and $\chi=m\left(u_{6}\right) / L$. They allow us to define the all-loop excitation densities through $\rho(u)=-\mathrm{d} \xi(u) / \mathrm{d} u$ and $\eta(w)=-\mathrm{d} \chi(w) / \mathrm{d} w$. One can show that this is consistent as the correct one-loop densities can be derived from the effective equations. 
Finally we take the derivatives wrt $u$ and $w$, respectively. Thus, (4.20) and (4.21) become:

$$
\begin{aligned}
\frac{1}{\mathrm{i}}\left(\frac{1}{\sqrt{u_{-}^{2}-4 g^{2}}}\right. & \left.-\frac{1}{\sqrt{u_{+}^{2}-4 g^{2}}}\right) \\
= & 2 \pi \rho(u)-2 \int_{-\infty}^{\infty} \frac{\rho\left(u^{\prime}\right) \mathrm{d} u^{\prime}}{\left(u-u^{\prime}\right)^{2}+1} \\
& +2 \mathrm{i} \int_{-\infty}^{\infty} \mathrm{d} u^{\prime} \rho\left(u^{\prime}\right) \frac{\mathrm{d}}{\mathrm{d} u}\left(\log \frac{1-g^{2} / x^{+}(u) x^{-}\left(u^{\prime}\right)}{1-g^{2} / x^{-}(u) x^{+}\left(u^{\prime}\right)}+\mathrm{i} \theta\left(u, u^{\prime}\right)\right) \\
& +2 \int_{-\infty}^{\infty} \frac{\eta(w) \mathrm{d} w}{(u-w)^{2}+1} \\
& +\mathrm{i} \int_{-\infty}^{\infty} \mathrm{d} w \eta(w) \frac{\mathrm{d}}{\mathrm{d} u}\left(\log \frac{1-g^{2} / x^{-}(u) x^{+}(w)}{1-g^{2} / x^{+}(u) x^{-}(w)}+\log \frac{1-g^{2} / x^{-}(u) x^{-}(w)}{1-g^{2} / x^{+}(u) x^{+}(w)}\right)
\end{aligned}
$$

and

$$
\begin{aligned}
0=2 \pi \eta(w)+ & 2 \int_{-\infty}^{\infty} \frac{\eta\left(w^{\prime}\right) \mathrm{d} w^{\prime}}{\left(w-w^{\prime}\right)^{2}+1}-2 \int_{-\infty}^{\infty} \frac{\rho(u) \mathrm{d} u}{(w-u)^{2}+1} \\
& +\mathrm{i} \int_{-\infty}^{\infty} \mathrm{d} u \rho(u) \frac{\mathrm{d}}{\mathrm{d} w}\left(\log \frac{1-g^{2} / x^{+}(w) x^{-}(u)}{1-g^{2} / x^{-}(w) x^{+}(u)}+\log \frac{1-g^{2} / x^{-}(w) x^{-}(u)}{1-g^{2} / x^{+}(w) x^{+}(u)}\right) .
\end{aligned}
$$

\subsection{All-loop energy}

Our strategy is now to solve (4.23) for the auxiliary root density $\eta$ as a functional of the energy-momentum-carrying main root density $\rho$. Substitution of this solution into (4.23) then yields a single, closed equation for the latter. This is easily done by Fourier transformation techniques, see in particular [15].

The Fourier transforms of the densities $\rho(u)$ and $\eta(w)$ are defined as

$$
\hat{\rho}(t)=\mathrm{e}^{-|t| / 2} \int_{-\infty}^{\infty} \mathrm{e}^{\mathrm{i} t u} \rho(u) \mathrm{d} u, \quad \hat{\eta}(t)=\mathrm{e}^{-|t| / 2} \int_{-\infty}^{\infty} \mathrm{e}^{\mathrm{i} t w} \eta(w) \mathrm{d} w
$$

where we have included a factor $\mathrm{e}^{-|t| / 2}$ for convenience. Including the same prefactors, the double Fourier transforms of the kernels

$$
\begin{aligned}
& K(w, u)=\frac{1}{2 \pi \mathrm{i}} \partial_{w} \log \frac{1-g^{2} / x^{+}(w) x^{-}(u)}{1-g^{2} / x^{-}(w) x^{+}(u)}, \\
& H(w, u)=\frac{1}{2 \pi \mathrm{i}} \partial_{w} \log \frac{1-g^{2} / x^{-}(w) x^{-}(u)}{1-g^{2} / x^{+}(w) x^{+}(u)},
\end{aligned}
$$

may be explicitly computed as, respectively,

$$
\begin{aligned}
& \hat{K}\left(t, t^{\prime}\right)=2 \pi g^{2}\left(1-\operatorname{sign} t t^{\prime}\right)|t| \mathrm{e}^{-|t|-\left|t^{\prime}\right|} \frac{J_{0}(2 g|t|) J_{1}\left(2 g\left|t^{\prime}\right|\right)-J_{0}\left(2 g\left|t^{\prime}\right|\right) J_{1}(2 g|t|)}{2 g\left(|t|-\left|t^{\prime}\right|\right)}, \\
& \hat{H}\left(t, t^{\prime}\right)=-2 \pi g^{2}\left(1+\operatorname{sign} t t^{\prime}\right)|t| \mathrm{e}^{-|t|-\left|t^{\prime}\right|} \frac{J_{0}(2 g|t|) J_{1}\left(2 g\left|t^{\prime}\right|\right)+J_{0}\left(2 g\left|t^{\prime}\right|\right) J_{1}(2 g|t|)}{2 g\left(|t|+\left|t^{\prime}\right|\right)} .
\end{aligned}
$$


Fourier transforming (4.23) diagonalizes all terms containing $\eta$, and one obtains

$$
\begin{aligned}
0=\mathrm{e}^{|t|} \hat{\eta}(t)+ & \hat{\eta}(t)-\hat{\rho}(t)-\frac{1}{2 \pi} \int_{-\infty}^{\infty} \mathrm{d} t^{\prime} \hat{\rho}\left(-t^{\prime}\right) \mathrm{e}^{|t|} \hat{K}\left(t, t^{\prime}\right) \mathrm{e}^{\left|t^{\prime}\right|} \\
& -\frac{1}{2 \pi} \int_{-\infty}^{\infty} \mathrm{d} t^{\prime} \hat{\rho}\left(-t^{\prime}\right) \mathrm{e}^{|t|} \hat{H}\left(t, t^{\prime}\right) \mathrm{e}^{\left|t^{\prime}\right|} .
\end{aligned}
$$

As announced above, we may now solve for $\hat{\eta}(t)$ in terms of an integral transform of $\hat{\rho}(t)$

$$
\hat{\eta}(t)=\frac{1}{\mathrm{e}^{t}+1}\left(\hat{\rho}(t)-4 g^{2} t \int_{0}^{\infty} \mathrm{d} t^{\prime} \hat{K}_{0}\left(2 g t, 2 g t^{\prime}\right) \hat{\rho}\left(t^{\prime}\right)\right)
$$

whose kernel $\hat{K}_{0}\left(t, t^{\prime}\right)$ is defined as in (1.3).

Now we treat (4.22) in a likewise fashion. The Fourier transform of the lhs is given by

$$
\frac{1}{2 \pi \mathrm{i}} \int_{-\infty}^{\infty} \mathrm{d} u\left(\frac{1}{\sqrt{u_{-}^{2}-4 g^{2}}}-\frac{1}{\sqrt{u_{+}^{2}-4 g^{2}}}\right) \mathrm{e}^{\mathrm{i} t u}=\mathrm{e}^{-|t| / 2} J_{0}(2 g t) .
$$

Thus, equation (4.22) reads in Fourier space

$$
\begin{aligned}
J_{0}(2 g t)=\mathrm{e}^{t} \hat{\rho}( & t)-\hat{\rho}(t)+\hat{\eta}(t) \\
& +4 g^{2} t \int_{0}^{\infty} \mathrm{d} t^{\prime}\left(\hat{K}_{m}\left(2 g t, 2 g t^{\prime}\right)+\hat{K}_{\mathrm{d}}\left(2 g t, 2 g t^{\prime}\right)\right) \hat{\rho}\left(t^{\prime}\right) \\
& -4 g^{2} t \int_{0}^{\infty} \mathrm{d} t^{\prime} \hat{K}_{1}\left(2 g t, 2 g t^{\prime}\right) \hat{\eta}\left(t^{\prime}\right),
\end{aligned}
$$

where we have used (1.1), and $\hat{K}_{1}$ is given in (1.4) while $\hat{K}_{m}=\hat{K}_{0}+\hat{K}_{1}$ is

$$
\hat{K}_{m}\left(t, t^{\prime}\right)=\frac{J_{1}(t) J_{0}\left(t^{\prime}\right)-J_{0}(t) J_{1}\left(t^{\prime}\right)}{t-t^{\prime}} .
$$

Now we may substitute (4.30) into (4.32). This leads to a single equation for $\hat{\rho}(t)$

$$
\begin{aligned}
\hat{\rho}(t)=\mathrm{e}^{-2 t}(1 & \left.+\mathrm{e}^{t}\right)\left(J_{0}(2 g t)\right. \\
& +4 g^{2} t \int_{0}^{\infty} \mathrm{d} t^{\prime}\left(\frac{1}{\mathrm{e}^{t}+1} \hat{K}_{0}\left(2 g t, 2 g t^{\prime}\right)+\frac{1}{\mathrm{e}^{t^{\prime}}+1} \hat{K}_{1}\left(2 g t, 2 g t^{\prime}\right)\right) \hat{\rho}\left(t^{\prime}\right) \\
& \left.-4 g^{2} t \int_{0}^{\infty} \mathrm{d} t^{\prime}\left(\hat{K}_{m}\left(2 g t, 2 g t^{\prime}\right)+\hat{K}_{\mathrm{n}}\left(2 g t, 2 g t^{\prime}\right)+\hat{K}_{\mathrm{d}}\left(2 g t, 2 g t^{\prime}\right)\right) \hat{\rho}\left(t^{\prime}\right)\right),
\end{aligned}
$$

as announced in (1.8) at the beginning of this paper. Since we have two degrees of freedom per unit length, i.e. the state is doubly filled, the density (4.34) is correspondingly normalized to 2 , i.e. $\hat{\rho}(0)=2$, see (4.24). The dressing kernel $\hat{K}_{\mathrm{d}}\left(t, t^{\prime}\right)$ is written in $(1.2)$, and we just proved that the nesting kernel $\hat{K}_{\mathrm{n}}\left(t, t^{\prime}\right)$ is indeed given by the structurally very similar expression (1.9). In fact, $2 \hat{K}_{\mathrm{n}}$ and $\hat{K}_{\mathrm{d}}$ differ only by a single sign in the denominator of the diagonal kernel $t /\left(\mathrm{e}^{t} \pm 1\right)$. 
The energy of the state can now be computed from the density by Fourier transforming (2.9). It reads

$$
E(g)=4 L \int_{0}^{\infty} \mathrm{d} t \frac{J_{1}(2 g t)}{2 g t} \hat{\rho}(t) .
$$

This leads via (2.8) to the anomalous dimension (1.7) announced at the beginning.

We expect our solution to be valid at arbitrary values of the coupling constant. The integral equation (4.34) is however (presumably) too complicated to be explicitly solvable. Being of Fredholm type, it is however very easy to expand in small $g$ to high orders. We thus find for the perturbative energy to e.g. seven-loop order

$$
\begin{aligned}
\frac{E(g)}{L}=3-\frac{51}{4} & g^{2}+\frac{393}{4} g^{4}-\left(\frac{59487}{64}+54 \zeta(3)\right) g^{6} \\
& +\left(\frac{632661}{64}+\frac{1665}{2} \zeta(3)+540 \zeta(5)\right) g^{8} \\
& -\left(\frac{29056443}{256}+\frac{87525}{8} \zeta(3)+8505 \zeta(5)+5670 \zeta(7)\right) g^{10} \\
& +\left(\frac{351914817}{256}+\frac{2244573}{16} \zeta(3)+972 \zeta(3)^{2}\right. \\
& +114723 \zeta(5)+90909 \zeta(7)+63504 \zeta(9)) g^{12}+\cdots
\end{aligned}
$$

We notice that zeta functions of odd, but not even, argument enter the energy. This is not surprising, as these are directly generated by the dressing kernel (1.1), cf [14]. If we assign a 'degree of transcendentality' $k$ to $\zeta(k)$ we see that the contributions at a given loop order are, in contradistinction to the case of large spin twist operators, see $[15,14,19]$, not of constant degree. One may nevertheless observe that at a given order $l$ the degree is bounded, and always saturated, by $2 l-5$. Note also that all zeta-function coefficients, as well as all rational numbers, turn out to be integers after factoring out inverse powers of 2 .

Conversely, the dressing factor is the only source of $\zeta$-function terms in the expansion (4.36). By this we mean that dropping the dressing factor from the asymptotic Bethe ansatz equations (2.7) would eradicate all terms containing $\zeta$-functions in (4.36), and would generate only rational loop contributions. The latter would precisely agree with all terms of 'transcendentality degree zero' in (4.36).

It is also interesting to note that the thermodynamic expansion of (4.36) does not coincide with the energies of finite length operators (see appendix $\mathrm{C}$ ), as opposed to the case of the pseudo-vacuum state $\operatorname{Tr} \mathcal{U}^{L}[31,33]$. By this we mean that the exact anomalous dimension of the operator $\operatorname{Tr} \mathcal{F}^{L}$, even below wrapping order, is not exactly proportional to $L$, while it is for $\operatorname{Tr} \mathcal{U}^{L}$. This is very likely due to the length changing processes starting for field strength operators at two-loop order, i.e. the number of fields in a local operator is not a conserved quantity at higher loops. Correspondingly, and in contradistinction to a BPS state $\operatorname{Tr} \mathcal{Z}^{L}$ or a fermionic pseudo-vacuum $\operatorname{Tr} \mathcal{U}^{L}$, the one-loop field strength operator $\operatorname{Tr} \mathcal{F}^{L}$ is not an exact eigenstate and will pick up higher order quantum corrections: $\operatorname{Tr} \mathcal{F}^{L}+\mathcal{O}\left(g^{2}\right)$. 


\section{Maximal filling and nesting}

We would like to demonstrate that the emergence of a nesting kernel (1.9), which we argue to be closely analogous to a dressing kernel (1.2), is a rather generic mechanism if two prerequisites are met. The first is that the states satisfy a special maximal filling condition, and the second is that the state is irreducibly ${ }^{5}$ nested.

Let us first study two simple cases where only the first, but not the second prerequisite is fulfilled. These are the 'highest energy' antiferromagnetic state of the $\mathfrak{s u}(2)$ sector [26,34], and the fermionic pseudo-vacuum state $\operatorname{Tr} \mathcal{U}^{L}[31,33]$. As a byproduct we will find the correct perturbative expansion of these states, as the expressions in $[26,34,31]$ were obtained with a trivial dressing factor $\sigma^{2}=1$, and need to be revised starting at four-loop order.

For the $\mathfrak{s u}(2)$ antiferromagnet the occupation numbers for an even length $L$ operator are

$$
\left(K_{1}, K_{2}, K_{3}, K_{4}, K_{5}, K_{6}, K_{7}\right)=\left(0,0,0, \frac{L}{2}, 0,0,0\right) .
$$

It is straightforward to repeat the analysis of $[26,34]$ in the presence of the non-trivial dressing phase (1.1), and one finds that the Fourier transformed density of roots satisfies in the thermodynamic limit the linear integral equation

$$
\hat{\rho}(t)=\frac{1}{\mathrm{e}^{t}+1}\left(J_{0}(2 g t)-4 g^{2} t \int_{0}^{\infty} \mathrm{d} t^{\prime} \hat{K}_{\mathrm{d}}\left(2 g t, 2 g t^{\prime}\right) \hat{\rho}\left(t^{\prime}\right)\right) .
$$

This is a half-filled state, which is the maximally possible filling in this sector. Correspondingly, the density is normalized to $1 / 2$, i.e. $\hat{\rho}(0)=1 / 2$, see $(4.24)$. The energy is as always given by (4.35). Dropping the convolution term on the rhs of (5.2) yields the density for the Lieb-Wu ground state energy of the fermionic Hubbard model in a closed form, see [26]. The $\mathfrak{s u}(2)$ sector of $\mathcal{N}=4$ gauge theory is a supersymmetric deformation of the latter. It is described by adding the backreacting convolution. Apparently, the density $\hat{\rho}(t)$ can no longer be found in closed form. However, it is interesting to work out the weak coupling expansion of our model from (4.35) and (5.2). One finds to the first few orders

$$
\begin{aligned}
\frac{E(g)}{L}=2 \zeta_{a}(1) & -6 \zeta_{a}(3) g^{2}+40 \zeta_{a}(5) g^{4}-\left(350 \zeta_{a}(7)+32 \zeta_{a}(1) \zeta_{a}(3) \zeta(3)\right) g^{6} \\
& +\left(3528 \zeta_{a}(9)+96 \zeta_{a}(3)^{2} \zeta(3)+320 \zeta_{a}(1) \zeta_{a}(5) \zeta(3)+320 \zeta_{a}(1) \zeta_{a}(3) \zeta(5)\right) g^{8} \\
& -\left(38808 \zeta_{a}(11)+1600 \zeta_{a}(3) \zeta_{a}(5) \zeta(3)+3360 \zeta_{a}(1) \zeta_{a}(7) \zeta(3)\right. \\
& \left.+1024 \zeta_{a}(3)^{2} \zeta(5)+3264 \zeta_{a}(1) \zeta_{a}(5) \zeta(5)+3360 \zeta_{a}(1) \zeta_{a}(3) \zeta(7)\right) g^{10} \\
& +\left(453024 \zeta_{a}(13)+6400 \zeta_{a}(5)^{2} \zeta(3)+15680 \zeta_{a}(3) \zeta_{a}(7) \zeta(3)\right. \\
& +37632 \zeta_{a}(1) \zeta_{a}(9) \zeta(3)+512 \zeta_{a}(1) \zeta_{a}(3)^{2} \zeta(3)^{2}+17792 \zeta_{a}(3) \zeta_{a}(5) \zeta(5) \\
& +34944 \zeta_{a}(1) \zeta_{a}(7) \zeta(5)+11200 \zeta_{a}(3)^{2} \zeta(7)+34944 \zeta_{a}(1) \zeta_{a}(5) \zeta(7) \\
& \left.+37632 \zeta_{a}(1) \zeta_{a}(3) \zeta(9)\right) g^{12}+\cdots .
\end{aligned}
$$

\footnotetext{
${ }^{5}$ By this we mean that the nesting cannot be removed by an exact dualization of the Bethe roots.
} 
Here, we have decided to distinguish the alternating 'fermionic' $\zeta_{a}$-function

$$
\zeta_{a}(k)=\frac{1}{\Gamma(k)} \int_{0}^{\infty} \frac{\mathrm{d} t}{t} \frac{t^{k}}{\mathrm{e}^{t}+1}=\sum_{n=1}^{\infty} \frac{(-1)^{n-1}}{n^{k}}
$$

from the ordinary 'bosonic' $\zeta$-function

$$
\zeta(k)=\frac{1}{\Gamma(k)} \int_{0}^{\infty} \frac{\mathrm{d} t}{t} \frac{t^{k}}{\mathrm{e}^{t}-1}=\sum_{n=1}^{\infty} \frac{1}{n^{k}},
$$

even though they are related by the formula $\zeta_{a}(k)=\left(1-2^{1-k}\right) \zeta(k)$. Note also that $\zeta_{a}(1)=\log (2)$. Using these relations we can 'simplify' the expression (5.3) to

$$
\begin{aligned}
\frac{E(g)}{L}=2 \log (2) & -\frac{9}{2} \zeta(3) g^{2}+\frac{75}{2} \zeta(5) g^{4}-\left(24 \log (2) \zeta(3)^{2}+\frac{11025}{32} \zeta(7)\right) g^{6} \\
+ & \left(54 \zeta(3)^{3}+540 \log (2) \zeta(3) \zeta(5)+\frac{112455}{32} \zeta(9)\right) g^{8} \\
& -\left(1701 \zeta(3)^{2} \zeta(5)+3060 \log (2) \zeta(5)^{2}+\frac{11655}{2} \log (2) \zeta(3) \zeta(7)\right. \\
& \left.+\frac{4962573}{128} \zeta(11)\right) g^{10} \\
+ & \left(288 \log (2) \zeta(3)^{4}+18135 \zeta(3) \zeta(5)^{2}\right. \\
+ & \frac{71505}{4} \zeta(3)^{2} \zeta(7)+67158 \log (2) \zeta(5) \zeta(7) \\
+ & \left.65709 \log (2) \zeta(3) \zeta(9)+\frac{57972915}{128} \zeta(13)\right) g^{12}+\cdots
\end{aligned}
$$

This however obscures the distinction between the contributions stemming from the dressing factor (the $\zeta$-terms) and from the fermionic Hubbard model (the $\zeta_{a}$-terms). In fact, omitting all terms containing $\zeta$ (in (5.3), but not in (5.6)!) leads back to the Hubbard ground state energy. This admixture of bosonic $\zeta$ and fermionic $\zeta_{a}$ is further evidence that planar AdS/CFT is a supersymmetric generalization of the purely fermionic Hubbard model as employed in [26]. Note also that the coefficients multiplying the $\zeta$ - and $\zeta_{a}$-functions in (5.3) are all integers. Interestingly, we see that the terms in (5.3) are still of constant degree of transcendentality $(2 l-1)$ at a given loop order $l$ if we assign a 'degree of transcendentality' $k$ to both $\zeta(k)$ and $\zeta_{a}(k)$.

We now turn to the $\mathfrak{s u}(1 \mid 1)$ state $\operatorname{Tr} \mathcal{U}^{L}$. The occupation numbers are

$$
\left(K_{1}, K_{2}, K_{3}, K_{4}, K_{5}, K_{6}, K_{7}\right)=(0,0,0, L, L-1,0,0) .
$$

In the picture of figure 1 , we first replace all $L$ fields $\mathcal{Z}$ in the BPS vacuum $\operatorname{Tr} \mathcal{Z}^{L}$ by $L$ bosons $\mathcal{X}$, and then turn the bosons into fermions $\mathcal{U}$. This state may be dualized, and the two-level nested Bethe equations may be converted to a single level [8]. Extending the analysis of [31] to the case of a non-trivial dressing phase (1.1), we find that the Fourier transformed density of roots satisfies in the limit $L \rightarrow \infty$ the equation

$\hat{\rho}(t)=\mathrm{e}^{-t}\left(J_{0}(2 g t)-2 g^{2} t \int_{0}^{\infty} \mathrm{d} t^{\prime}\left(\hat{K}_{m}\left(2 g t, 2 g t^{\prime}\right)+2 \hat{K}_{\mathrm{d}}\left(2 g t, 2 g t^{\prime}\right)\right) \hat{\rho}\left(t^{\prime}\right)\right)$. 
This is a filled state, with one excitation per lattice site, which is the maximally possible filling in this sector. Correspondingly, the density is normalized to 1 , i.e. $\hat{\rho}(0)=1$. Working out the weak coupling expansion of this state from (4.35) and (5.8), one finds to e.g. eight-loop order

$$
\begin{aligned}
\frac{E(g)}{L}=2-8 & g^{2}+58 g^{4}-(518+32 \zeta(3)) g^{6}+(5228+480 \zeta(3)+320 \zeta(5)) g^{8} \\
& -(57280+6144 \zeta(3)+4928 \zeta(5)+3360 \zeta(7)) g^{10} \\
& +\left(665344+76768 \zeta(3)+512 \zeta(3)^{2}+64960 \zeta(5)\right. \\
& +52864 \zeta(7)+37632 \zeta(9)) g^{12} \\
& -\left(8070352+965856 \zeta(3)+13312 \zeta(3)^{2}+833792 \zeta(5)+10240 \zeta(3) \zeta(5)\right. \\
& +713056 \zeta(7)+602112 \zeta(9)+443520 \zeta(11)) g^{14}+\cdots
\end{aligned}
$$

The $\zeta$-functions are exclusively generated by the dressing factor. We note similar 'transcendentality properties' as in the $\operatorname{Tr} \mathcal{F}^{L}$ case of the last section, namely that at a given loop order $l$ combinations of zeta functions with odd arguments occur up to and including degree of transcendentality $2 l-5$. Again all zeta-function coefficients, as well as all rational numbers, turn out to be integers.

Let us finally present a third example, namely a certain $\mathfrak{s o}(6)$ singlet state. At oneloop order this state is the highest energy state of a $\mathfrak{s o}(6)$ magnet $[1,35]$. At higher loops, however, the $\mathfrak{s o}(6)$ subsector is not closed anymore and thus one is forced to use the full $\mathfrak{p} \mathfrak{s u}(2,2 \mid 4)$ Bethe equations. The excitation scheme for this state reads

$$
\left(K_{1}, K_{2}, K_{3}, K_{4}, K_{5}, K_{6}, K_{7}\right)=\left(\frac{L}{2}-2, \frac{L}{2}-1, \frac{L}{2}, L, \frac{L}{2}, \frac{L}{2}-1, \frac{L}{2}-2\right) \text {. }
$$

After the dualization of the $u_{5}$ and $u_{3}$ roots one is left with the following equations

$$
\begin{aligned}
& 1=\prod_{\substack{j=1 \\
j \neq k}}^{L / 2-1} \frac{u_{2, k}-u_{2, j}+\mathrm{i}}{u_{2, k}-u_{2, j}-\mathrm{i}} \prod_{j=1}^{L-2} \frac{u_{2, k}-\tilde{u}_{3, j}-\mathrm{i} / 2}{u_{2, k}-\tilde{u}_{3, j}+\mathrm{i} / 2} \\
& 1=\prod_{j=1}^{L / 2-1} \frac{\tilde{u}_{3, k}-u_{2, j}+\mathrm{i} / 2}{\tilde{u}_{3, k}-u_{2, j}-\mathrm{i} / 2} \prod_{j=1}^{L} \frac{\tilde{x}_{3, k}-x_{4, j}^{+}}{\tilde{x}_{3, k}-x_{4, j}^{-}} \\
& \left(\frac{x_{4, k}^{+}}{x_{4, k}^{-}}\right)^{L}=\prod_{j=1}^{L} \frac{x_{4, k}^{-}-x_{4, j}^{+}}{x_{4, k}^{+}-x_{4, j}^{-}} \frac{1-g^{2} / x_{4, k}^{+} x_{4, j}^{-}}{1-g^{2} / x_{4, k}^{-} x_{4, j}^{+}} \sigma^{2}\left(u_{4, k}, u_{4, j}\right) \prod_{j=1}^{L-2} \frac{x_{4, k}^{+}-\tilde{x}_{3, j}}{x_{4, k}^{-}-\tilde{x}_{3, j}} \prod_{j=1}^{L-2} \frac{x_{4, k}^{+}-\tilde{x}_{5, j}}{x_{4, k}^{-}-\tilde{x}_{5, j}} \\
& 1=\prod_{\substack{j=1 \\
j \neq k}}^{L} \frac{\tilde{u}_{5, k}-u_{6, j}+\mathrm{i} / 2}{\tilde{u}_{5, k}-u_{6, j}-\mathrm{i} / 2} \prod_{j=1}^{L} \frac{\tilde{x}_{5, k}-x_{4, j}^{+}}{\tilde{x}_{5, k}-x_{4, j}^{-}} \\
& 1=\prod_{\substack{j=1 \\
j \neq k}}^{L-1} \frac{u_{6, k}-u_{6, j}+\mathrm{i}}{u_{6, k}-u_{6, j}-\mathrm{i}} \prod_{j=1}^{L-2} \frac{u_{6, k}-\tilde{u}_{5, j}-\mathrm{i} / 2}{u_{6, k}-\tilde{u}_{5, j}+\mathrm{i} / 2} .
\end{aligned}
$$


For the highest energy state we may assume $u_{2, j}=u_{6, j}$ and $u_{3, k}=u_{5, k}$ for $j=$ $1, \ldots, L / 2-1$ and $k=1, \ldots, L-2$, respectively. After this is done one notes a striking structural similarity to (3.12)-(3.14). Apart from the different overall number of magnons the resulting equations differ only by the power of the interaction term in the equation for the $u_{4}$ roots. It is thus plausible to assume that the $u_{5}$ and $u_{6}$ roots will again form stacks, even though this seems to be more difficult to prove in this case. Under this assumption, the derivation of the integral equation for the principal density is straightforward and follows the same lines as above. In Fourier space one now gets, in great similarity to (1.8),

$$
\begin{aligned}
\hat{\rho}(t)=\frac{\mathrm{e}^{t}+1}{\mathrm{e}^{2 t}+1} & \left(J_{0}(2 g t)\right. \\
& -4 g^{2} t \int_{0}^{\infty} \mathrm{d} t^{\prime}\left(\frac{\mathrm{e}^{t}-1}{\mathrm{e}^{t}+1} \hat{K}_{0}\left(2 g t, 2 g t^{\prime}\right)+\frac{\mathrm{e}^{t^{\prime}}-1}{\mathrm{e}^{t^{\prime}}+1} \hat{K}_{1}\left(2 g t, 2 g t^{\prime}\right)\right) \hat{\rho}\left(t^{\prime}\right) \\
& \left.-4 g^{2} t \int_{0}^{\infty} \mathrm{d} t^{\prime}\left(2 \hat{K}_{\mathrm{n}}\left(2 g t, 2 g t^{\prime}\right)+\hat{K}_{\mathrm{d}}\left(2 g t, 2 g t^{\prime}\right)\right) \hat{\rho}\left(t^{\prime}\right)\right) .
\end{aligned}
$$

Interestingly, the very same nesting kernel $\hat{K}_{\mathrm{n}}(1.9)$ appears, with an overall factor of two, as in the case of the field strength operator, cf (1.8). This is precisely what one should expect.

It is again rather straightforward to find the weak coupling expansion of the energy to, say, four-loop order (we have highlighted the terms generated by the dressing phase)

$$
\begin{aligned}
\frac{E(g)}{L}=2 \beta(1) & +\zeta_{a}(1) \\
& -\left(4 \beta(1) \beta(2)+6 \beta(3)+2 \beta(2) \zeta_{a}(1)-\beta(1) \zeta_{a}(2)\right. \\
& \left.-\frac{1}{2} \zeta_{a}(1) \zeta_{a}(2)+\frac{3}{4} \zeta_{a}(3)\right) g^{2} \\
& +\left(8 \beta(1) \beta(2)^{2}+8 \beta(2) \beta(3)+24 \beta(1) \beta(4)+40 \beta(5)+4 \beta(2)^{2} \zeta_{a}(1)\right. \\
& +12 \beta(4) \zeta_{a}(1)-4 \beta(1) \beta(2) \zeta_{a}(2)-2 \beta(3) \zeta_{a}(2)-2 \beta(2) \zeta_{a}(1) \zeta_{a}(2) \\
& +\frac{1}{2} \beta(1) \zeta_{a}(2)^{2}+\frac{1}{4} \zeta_{a}(1) \zeta_{a}(2)^{2}+\beta(2) \zeta_{a}(3)-\frac{1}{4} \zeta_{a}(2) \zeta_{a}(3)-\frac{3}{2} \beta(1) \zeta_{a}(4) \\
& \left.-\frac{3}{4} \zeta_{a}(1) \zeta_{a}(4)+\frac{5}{4} \zeta_{a}(5)\right) g^{4} \\
& -\left(16 \beta(1) \beta(2)^{3}+16 \beta(2)^{2} \beta(3)+16 \beta(1) \beta(3)^{2}\right. \\
& +96 \beta(1) \beta(2) \beta(4)+44 \beta(3) \beta(4) \\
& +40 \beta(2) \beta(5)+200 \beta(1) \beta(6)+350 \beta(7) \\
& +\mathbf{3 2} \boldsymbol{\beta}(\mathbf{1}) \boldsymbol{\beta}(\mathbf{3}) \boldsymbol{\zeta}(\mathbf{3})+8 \beta(2)^{3} \zeta_{a}(1) \\
& +8 \beta(3)^{2} \zeta_{a}(1)+48 \beta(2) \beta(4) \zeta_{a}(1)+100 \beta(6) \zeta_{a}(1)+\mathbf{1 6} \boldsymbol{\beta}(\mathbf{3}) \boldsymbol{\zeta}_{\boldsymbol{a}}(\mathbf{1}) \boldsymbol{\zeta}(\mathbf{3}) \\
& -12 \beta(1) \beta(2)^{2} \zeta_{a}(2)-8 \beta(2) \beta(3) \zeta_{a}(2)-24 \beta(1) \beta(4) \zeta_{a}(2)-10 \beta(5) \zeta_{a}(2) \\
& -6 \beta(2)^{2} \zeta_{a}(1) \zeta_{a}(2)-12 \beta(4) \zeta_{a}(1) \zeta_{a}(2)+3 \beta(1) \beta(2) \zeta_{a}(2)^{2}+\beta(3) \zeta_{a}(2)^{2} \\
& +\frac{3}{2} \beta(2) \zeta_{a}(1) \zeta_{a}(2)^{2}-\frac{1}{4} \beta(1) \zeta_{a}(2)^{3}-\frac{1}{8} \zeta_{a}(1) \zeta_{a}(2)^{3}+2 \beta(2)^{2} \zeta_{a}(3) \\
& +4 \beta(1) \beta(3) \zeta_{a}(3)+\frac{11}{2} \beta(4) \zeta_{a}(3)+\mathbf{4} \boldsymbol{\beta}(\mathbf{1}) \boldsymbol{\zeta}_{\boldsymbol{a}}(\mathbf{3}) \boldsymbol{\zeta}(\mathbf{3})+2 \beta(3) \zeta_{a}(1) \zeta_{a}(3) \\
& +\mathbf{2} \boldsymbol{\zeta}_{a}(\mathbf{1}) \boldsymbol{\zeta}_{\boldsymbol{a}}(\mathbf{3}) \boldsymbol{\zeta}(\mathbf{3})-\beta(2) \zeta_{a}(2) \zeta_{a}(3)+\frac{1}{8} \zeta_{a}(2)^{2} \zeta_{a}(3)+\frac{1}{4} \beta(1) \zeta_{a}(3)^{2} \\
& -
\end{aligned}
$$




$$
\begin{aligned}
& +\frac{1}{8} \zeta_{a}(1) \zeta_{a}(3)^{2}-6 \beta(1) \beta(2) \zeta_{a}(4)-\frac{11}{4} \beta(3) \zeta_{a}(4)-3 \beta(2) \zeta_{a}(1) \zeta_{a}(4) \\
& +\frac{3}{2} \beta(1) \zeta_{a}(2) \zeta_{a}(4)+\frac{3}{4} \zeta_{a}(1) \zeta_{a}(2) \zeta_{a}(4)-\frac{11}{32} \zeta_{a}(3) \zeta_{a}(4)+\frac{5}{4} \beta(2) \zeta_{a}(5) \\
& \left.-\frac{5}{16} \zeta_{a}(2) \zeta_{a}(5)-\frac{25}{8} \beta(1) \zeta_{a}(6)-\frac{25}{16} \zeta_{a}(1) \zeta_{a}(6)+\frac{175}{64} \zeta_{a}(7)\right) g^{6}+\cdots
\end{aligned}
$$

For this state, an interesting new set of numbers appears, namely the Dirichlet $\beta$-function evaluated at positive integers:

$$
\beta(k)=\frac{1}{\Gamma(k)} \int_{0}^{\infty} \frac{\mathrm{d} t}{t} \frac{t^{k} \mathrm{e}^{t}}{\mathrm{e}^{2 t}+1}=\sum_{n=0}^{\infty} \frac{(-1)^{n}}{(2 n+1)^{k}} .
$$

If $k$ is odd this leads to $\pi^{k}$ times rational numbers (related to Euler numbers). If $k$ is even the numbers $\beta(k)$ cannot be expressed through $\pi$ 's. $\beta(2)$ is Catalan's constant. All coefficients multiplying the products of $\zeta_{-}, \zeta_{a}$, and $\beta$-functions are integers after factoring out inverse powers of 2 .

Note that (5.12) is the higher loop generalization of a one-loop result worked out in [1]. The latter is of course reproduced from (5.13) since $\beta(1)=\pi / 4$ and $\zeta_{a}(1)=\log (2)$. Note that this state's energy satisfies, just as the half-filled $\mathfrak{s u}(2)$ state's energy (5.3), a constant transcendentality principle.

\section{Outlook}

We have shown in detail how to compute the anomalous dimension of the field strength pseudo-vacuum (1.6) in the thermodynamic limit from the nested asymptotic Bethe equations of [8]. Several techniques to reduce the number of equations were introduced and a single effective integral equation (1.8) for the distribution density of Bethe roots was derived from a starting set of five. Combining this equation with the expression (1.7) relating the density to the operator dimension, it is straightforward to find the weak coupling expansion of the latter to any desired order, cf (4.36). Incidentally, as our equation is analytic in the vicinity of $g=0$, it should also be, by analytic continuation, just as valid at any value of the coupling. It would be very interesting to analyse it in the strong coupling limit $g \rightarrow \infty$, and to interpret the corresponding state in string theory. The techniques developed in [36] might be useful here.

Interestingly, the influence of the nesting on the effective Bethe equation results in a kernel (1.9) which strongly resembles the dressing kernel (1.2) recently proposed in [14]. This leads us to suggest that the dressing phase should originate from the elimination of further, yet to be found auxiliary Bethe roots. A formal procedure, unfortunately plagued with difficulties, is briefly discussed in appendix D (see also [37]). The detailed mechanism for how this happens therefore remains to be worked out. The techniques presented in the present paper might prove helpful in this respect.

We would also like to stress that 'extra Bethe ansatz levels' in order to improve the asymptotic equations of [8] have been proposed previously. These are natural from general arguments concerning finite size effects [38], from concrete indications that the asymptotic Bethe ansatz needs to be corrected [39], and finally from studies indicating that the BDS/Hubbard magnon dispersion law at strong coupling [40] is to be corrected in a finite volume [41] (see also [42] and [43]). In particular, Hubbard-type models are able to create long-range integrable systems from short-range interactions [26] (see [44] 
for a detailed study on the relation of the nested to the effective Bethe equations near the antiferromagnetic vacuum). Furthermore, similar mechanisms have also been proposed on the level of the string sigma model $[27,28]$. It is interesting to note that one key feature of [26]-[28] is that the BPS vacuum corresponds to a non-trivial distribution of top level Bethe roots. This qualitatively agrees with our result, which suggests that the 'physical' BPS vacuum states (1.5) are created by filling up a truly empty 'unphysical' reference state. See also the closely related comments in [45].

The number of hidden Bethe roots creating the dressing factor of [14] is infinite if the ansatz is to be asymptotically exact for short operators. This should be related to the non-compact nature of the AdS/CFT system. In such a situation Bethe equations might not necessarily furnish the most effective description, and an approach based on Sklyanin's separation-of-variables technique might be more appropriate. This would then replace the Bethe equations by functional equations for a nested set of Baxter $Q$-functions. Some of the latter should be non-polynomial in nature. Possibly the techniques used in $[46,47]$ might be useful in this context.

\section{Acknowledgments}

We would like to thank Niklas Beisert, Burkhard Eden, Lisa Freyhult, Sergey Frolov, Didina Serban, Jörg Teschner and Arkady Tseytlin for useful discussions.

\section{Appendix A. Properties of the generating polynomials}

In this section we will analyse some properties of the generating polynomials $Q_{4}$ and $Q_{6}$. The mathematical description of the formation of stacks will be given in the next section.

Let us start with the following observation: if $Q(x)$ is a polynomial with only real roots then

$$
S(x)=Q(x+\mathrm{i} s)+\alpha Q(x-\mathrm{i} s)
$$

with $|\alpha|=1$ also has only real roots. To prove this one observes, that for any real $y$

$$
|Q(x+\mathrm{i} y)|=|Q(x-\mathrm{i} y)|=\prod_{i=1}^{n}\left|\left(x-w_{j}\right)+\mathrm{i} y\right|
$$

grows monotonically with increasing $|y|$. Furthermore, if $x_{j}$ satisfies:

$$
S\left(x_{j}\right)=Q\left(x_{j}+\mathrm{i} s\right)+\alpha Q\left(x_{j}-\mathrm{i} s\right)=0
$$

then $\left|Q\left(x_{j}+\mathrm{i} s\right)\right|=\left|Q\left(x_{j}-\mathrm{i} s\right)\right|$. Together with the previous observation this implies $x_{j} \in \mathbb{R}$. Applying this theorem twice to $w^{L}$ one proves immediately that $Q_{6}(w)$ has only real roots. Similarly

$$
Q_{4}(u)=W\left(u+\frac{\mathrm{i}}{2}\right)-W\left(u-\frac{\mathrm{i}}{2}\right), \quad W(u)=u^{L}\left((u+\mathrm{i})^{L}-(u-\mathrm{i})^{L}\right)
$$

also has only real roots. If one orders the roots of $Q(x)$ as follows $x_{1} \leq x_{2} \leq \cdots \leq x_{\mathrm{n}}$ one finds that

$$
Q^{\prime}\left(x_{i}\right) Q^{\prime}\left(x_{i+1}\right) \leq 0 .
$$


Thus, either $x_{i}$ or $x_{i+1}$ are zeros of $Q^{\prime}(x)$ or there exist a $\theta$ with $0<\theta<1$ such that

$$
Q^{\prime}\left(x_{i}+\theta\left(x_{i+1}-x_{i}\right)\right)=0, \quad i=1, \ldots, n-1 .
$$

In case of $Q_{6}(w)$ one finds

$$
\frac{\mathrm{d}}{\mathrm{d} w} Q_{6}(w, L)=L Q_{6}(w, L-1) .
$$

Thus, between any two zeros of $Q_{6}(w, L)$ there lies a zero of $Q_{6}(w, L-1)$. Furthermore it is fairly easy to prove that the resultant (given by the determinant of the corresponding Sylvester matrix) of $Q_{6}(w, L)$ and $Q_{6}(w, L-1)$ is always non-vanishing. Finally, it is possible to find an approximate formula for the extreme roots of $Q_{6}(w, L)$. Expecting this roots to scale with $L$ we set

$$
w_{\max }= \pm a L \text {. }
$$

Using

$$
\lim _{L \rightarrow \infty}\left(1+\frac{c}{L}\right)^{L}=\mathrm{e}^{c}
$$

we write

$$
Q_{6}( \pm a L, L)=-8( \pm a L)^{L} \cos \left(\frac{ \pm 1}{2 a}\right) \sin \left(\frac{ \pm 1}{2 a}\right)^{2}+\mathcal{O}\left(\frac{1}{L}\right) .
$$

Setting $a=1 / \pi$ one finds that $Q_{6}((1 / \pi) L+\epsilon, L)$ changes sign with $\epsilon$ and hence,

$$
w_{\max } \simeq \pm \frac{1}{\pi} L, \quad L \gg 1 .
$$

We conclude that for any $L>2$ the generating polynomial $Q_{6}(w, L)$ has $(L-2)$ distinct real roots, forming a dense set in $\mathbb{R}$ for $L \rightarrow \infty$. Curiously $Q_{6}(w, L)$ is the polynomial solution of

$$
Q_{6}\left(w+\frac{3 \mathrm{i}}{2}\right)+Q_{6}\left(w-\frac{3 \mathrm{i}}{2}\right)+Q_{6}\left(w+\frac{\mathrm{i}}{2}\right)+Q_{6}\left(w-\frac{\mathrm{i}}{2}\right)=2^{L} Q_{6}\left(\frac{w}{2}\right) .
$$

This should be interpreted as the Baxter equation for the $u_{6}$ roots. Similarly one can find extreme roots of $Q_{4}(u, L)$ and $Q_{5}(v, L)$. Surprisingly they are also given by (A.1).

\section{Appendix B. Formation of stacks}

Extreme roots of $Q_{5}$ are indeed real (as demonstrated above). In this section we will, however, prove that almost all roots of $Q_{5}(v, L)$, for large values of $\mathrm{L}$, occupy two contours shifted from the real axis by $\pm \mathrm{i} / 2$, respectively. In other words we will show that

$$
Q_{5}(x, L)=Q_{6}\left(x+\frac{\mathrm{i}}{2}, L\right) Q_{6}\left(x-\frac{\mathrm{i}}{2}, L\right)+\mathcal{O}\left(\frac{1}{L}\right)
$$

where $\mathcal{O}(1 / L)$ can be neglected in the large $L$ limit. 


\section{B.1. Power expansion of the polynomials}

After some computation it is possible to find for the polynomial $Q_{5}(v)$

$$
\begin{aligned}
Q_{5}(v)=3 v^{2 L} & -2 \sum_{k=0}^{L}\left(\begin{array}{l}
L \\
k
\end{array}\right) v^{2 L-k} \cos \frac{\pi k}{2}-4 \sum_{k=0}^{L} 2^{k}\left(\begin{array}{l}
L \\
k
\end{array}\right) v^{2 L-k} \cos \frac{\pi k}{2} \\
& +2 \sum_{k=0}^{L}\left(\begin{array}{l}
L \\
k
\end{array}\right) v^{2 L-k} \cos \frac{\pi k}{2}{ }_{2} F_{1}[-L,-k ; 1+L-k ; 2] \\
& +2 \sum_{k=L+1}^{2 L}\left(\begin{array}{c}
L \\
2 L-k
\end{array}\right) 2^{k-L} v^{2 L-k} \cos \frac{\pi k}{2}{ }_{2} F_{1}[-L, k-2 L ; 1+k-L ; 2] \\
& +\sum_{k=0}^{L}\left(\begin{array}{l}
L \\
k
\end{array}\right) v^{2(L-k)} 4^{k} .
\end{aligned}
$$

Thus one finds immediately that

$$
Q_{5}(v)=\sum_{n=0}^{2 L-4} c_{\mathrm{n}} v^{n}
$$

where the coefficients are given by

$$
\begin{aligned}
c_{\mathrm{n}}=-2\left(\begin{array}{c}
L \\
2 L
\end{array}\right. & -n) \cos \frac{\pi(2 L-n)}{2}-42^{2 L-n}\left(\begin{array}{c}
L \\
2 L-n
\end{array}\right) \cos \frac{\pi(2 L-n)}{2} \\
& +2\left(\begin{array}{c}
L \\
2 L-n
\end{array}\right) \cos \frac{\pi(2 L-n)}{2}{ }_{2} F_{1}[-L, n-2 L ; 1+n-L ; 2] \\
& +\left(\begin{array}{c}
L \\
n / 2
\end{array}\right) \cos ^{2}\left(\frac{\pi n}{2}\right) 4^{L-n / 2}
\end{aligned}
$$

for $n=L, L+1, \ldots, 2 L-4$ and

$$
\begin{aligned}
c_{\mathrm{n}}=-2\left(\begin{array}{c}
L \\
2 L
\end{array}\right. & -n) \cos \frac{\pi(2 L-n)}{2}-42^{2 L-n}\left(\begin{array}{c}
L \\
2 L-n
\end{array}\right) \cos \frac{\pi(2 L-n)}{2} \\
& +2\left(\begin{array}{c}
L \\
n
\end{array}\right) 2^{L-n} \cos \frac{\pi(2 L-n)}{2}{ }_{2} F_{1}[-L,-n ; 1+L-n ; 2] \\
& +\left(\begin{array}{c}
L \\
n / 2
\end{array}\right) \cos ^{2}\left(\frac{\pi n}{2}\right) 4^{L-n / 2}
\end{aligned}
$$

for $n=0, \ldots, L-1$. Similarly one finds

$$
\begin{aligned}
Q_{6}\left(w+\frac{\mathrm{i}}{2}\right) & Q_{6}\left(w-\frac{\mathrm{i}}{2}\right) \\
= & w^{2 L}-2 \sum_{k=0}^{2 L}\left(\begin{array}{c}
2 L \\
k
\end{array}\right) w^{2 L-k} \cos \frac{\pi k}{2}
\end{aligned}
$$




$$
\begin{aligned}
& -2 \sum_{k=0}^{L} 2^{k}\left(\begin{array}{l}
L \\
k
\end{array}\right) w^{2 L-k} \cos \frac{\pi k}{2} \\
& +2 \sum_{k=0}^{L}\left(\begin{array}{l}
L \\
k
\end{array}\right) w^{2(L-k)}+\sum_{k=0}^{L}\left(\begin{array}{l}
L \\
k
\end{array}\right) w^{2(L-k)} 4^{k} \\
& +2 \sum_{k=0}^{L} w^{2 L-k}\left(\begin{array}{l}
L \\
k
\end{array}\right) \cos \frac{\pi k}{2}\left({ }_{2} F_{1}[-L,-k ; 1+L-k ; 2]\right. \\
& \left.-{ }_{2} F_{1}[-L,-k ; 1+L-k ;-2]\right) \\
& +2 \sum_{k=L+1}^{2 L} w^{2 L-k} 2^{k-L}\left(\begin{array}{c}
L \\
2 L-k
\end{array}\right) \cos \frac{\pi k}{2}\left({ }_{2} F_{1}[-L, k-2 L ; 1+k-L ; 2]\right. \\
& \left.-{ }_{2} F_{1}[-L, k-2 L ; 1+k-L ;-2]\right) .
\end{aligned}
$$

The corresponding coefficients in the power expansion of

$$
Q_{6}\left(w+\frac{\mathrm{i}}{2}\right) Q_{6}\left(w-\frac{\mathrm{i}}{2}\right)=\sum_{n=0}^{2 L-4} d_{\mathrm{n}} w^{n}
$$

can easily be read off

$$
\begin{aligned}
d_{\mathrm{n}}=-2\left(\begin{array}{c}
2 L \\
n
\end{array}\right) \cos \frac{\pi(2 L-n)}{2}-22^{2 L-n}\left(\begin{array}{c}
L \\
2 L-n
\end{array}\right) \cos \frac{\pi(2 L-n)}{2} \\
+2\left(\begin{array}{c}
L \\
n / 2
\end{array}\right) \cos ^{2}\left(\frac{\pi n}{2}\right)+\left(\begin{array}{c}
L \\
n / 2
\end{array}\right) 4^{L-n / 2} \cos ^{2}\left(\frac{\pi n}{2}\right) \\
+2\left(\begin{array}{c}
L \\
2 L-n
\end{array}\right) \cos \frac{\pi(2 L-n)}{2}\left({ }_{2} F_{1}[-L, n-2 L ; 1+n-L ; 2]\right. \\
\left.-{ }_{2} F_{1}[-L, n-2 L ; 1+n-L ;-2]\right)
\end{aligned}
$$

for $n=L, \ldots, 2 L-4$ and

$$
\begin{aligned}
d_{\mathrm{n}}=-2\left(\begin{array}{c}
2 L \\
n
\end{array}\right) & \cos \frac{\pi(2 L-n)}{2}-22^{2 L-n}\left(\begin{array}{c}
L \\
2 L-n
\end{array}\right) \cos \frac{\pi(2 L-n)}{2} \\
& +2\left(\begin{array}{c}
L \\
n / 2
\end{array}\right) \cos ^{2}\left(\frac{\pi n}{2}\right)+\left(\begin{array}{c}
L \\
n / 2
\end{array}\right) 4^{L-n / 2} \cos ^{2}\left(\frac{\pi n}{2}\right) \\
& +22^{L-n}\left(\begin{array}{c}
L \\
n
\end{array}\right) \cos \frac{\pi(2 L-n)}{2}\left({ }_{2} F_{1}[-L,-n ; 1+L-n ; 2]\right. \\
& \left.-(-1)^{L-n} F_{1}[-L,-n ; 1+L-n ;-2]\right)
\end{aligned}
$$

for $n=0,1, \ldots, L-1$.

\section{B.2. Emergence of the stack picture}

In this subsection we assume $n \geq L$ ( $n<L$ can be analysed analogously). Furthermore without loss of generality we consider $L$ and $n$ to be even. The non-zero coefficients are 
then given by

$$
\begin{aligned}
& c_{\mathrm{n}}(L)=2(-1)^{n / 2}\left(\begin{array}{c}
L \\
2 L-n
\end{array}\right)\left({ }_{2} F_{1}[-L, n-2 L ; 1+n-L ; 2]-1-22^{2 L-n}\right) \\
&+\left(\begin{array}{c}
L \\
n / 2
\end{array}\right) 4^{L-n / 2} \\
& d_{\mathrm{n}}(L)=2(-1)^{n / 2}\left(\begin{array}{c}
L \\
2 L-n
\end{array}\right)\left({ }_{2} F_{1}[-L, n-2 L ; 1+n-L ; 2]\right. \\
&\left.-{ }_{2} F_{1}[-L, n-2 L ; 1+n-L ;-2]-2^{2 L-n}\right) \\
&-2(-1)^{n / 2}\left(\begin{array}{c}
2 L \\
n
\end{array}\right)+\left(\begin{array}{c}
L \\
n / 2
\end{array}\right)\left(2+4^{L-n / 2}\right) .
\end{aligned}
$$

B.2.1. $n=2 L-a$ case. The case $n=2 L-a$ with $a \geq 4$ and finite $a$ is fairly easy to analyse. Noting that

$\frac{(-L)_{k}}{(1+L-a)_{k}}=(-1)^{k}\left(1+\frac{a k(1-k)}{L}+\frac{1}{2 L^{2}}(a-k) k\left(-1+a+a k-k^{2}\right)+\cdots\right)$

one can immediately shown that for the large $L$ expansion of ${ }_{2} F_{1}[-L,-a ; 1+L-a ; \pm 2]$

${ }_{2} F_{1}[-L,-a ; 1+L-a ; 2]=3^{a}\left(1+\frac{2}{9 L} a(a-1)+\frac{2}{81 L^{2}} a\left(a^{3}+3 a^{2}-7 a+3\right)+\cdots\right)$

${ }_{2} F_{1}[-L,-a ; 1+L-a ;-2]=(-1)^{a}\left(1-\frac{2}{L} a(a-1)+\frac{2}{L^{2}} a\left(a^{3}-7 a^{2}+13 a-7\right)+\cdots\right)$.

Expanding $c_{\mathrm{n}}$ and $d_{\mathrm{n}}$ we note that

$$
\frac{c_{\mathrm{n}}(L)}{d_{\mathrm{n}}(L)}=1+\mathcal{O}\left(\frac{1}{L}\right)
$$

for large values of $L$.

B.2.2. Other values of $n$. Other values of $n$ are much more difficult to analyse since their generic dependence on $L$ is

$$
n=\alpha L+\beta, \quad 1 \leq \alpha<2
$$

and the summation limit of ${ }_{2} F_{1}$ depends now on $L$. Here we will show that $c_{\mathrm{n}} / d_{\mathrm{n}}-1$ is at least of order $\mathcal{O}(1 / L)$. We start with noting that for this values of $n$ one has

$$
{ }_{2} F_{1}[-L, n-2 L ; 1+n-L ; 2]>1+2^{2 L-n}+\frac{L(n-2 L)}{L-n-1}\left(2+2^{2 L-n-1}\right)+\cdots
$$

i.e. terms of the form $\left(\begin{array}{c}L \\ 2 L-n\end{array}\right) 2^{2 L-n}$ are least of all subleading. Next we observe that

$$
\begin{aligned}
\left(\begin{array}{c}
L \\
2 L-n
\end{array}\right) & { }_{2} F_{1}[-L, n-2 L ; 1+n-L ; 2] \\
= & \sum_{j=0}^{L}\left(\begin{array}{c}
L \\
j
\end{array}\right)\left(\begin{array}{c}
L \\
2 L-n-j
\end{array}\right) 2^{j} \\
& >\sum_{j=0}^{L}\left(\begin{array}{c}
L \\
j
\end{array}\right)\left(\begin{array}{c}
L \\
2 L-n-j
\end{array}\right) j=L \frac{n}{2 L}\left(\begin{array}{c}
2 L \\
n
\end{array}\right) \simeq \alpha L\left(\begin{array}{c}
2 L \\
n
\end{array}\right)+\mathcal{O}\left(\frac{1}{L}\right) .
\end{aligned}
$$


This implies that the $\left(\begin{array}{c}2 L \\ n\end{array}\right)$ term in (B.9) is at least subleading. Let us define the function

$$
f_{2 L-n, L}(x)=\sum_{j=0}^{L}\left(\begin{array}{c}
L \\
j
\end{array}\right)\left(\begin{array}{c}
L \\
2 L-n-j
\end{array}\right) x^{j}=P_{\tilde{n}}^{(L-\tilde{n},-2 L-1)}(1-2 x)
$$

where $P_{m}^{(\alpha, \beta)}(x)$ stands for the Jacobi polynomials (see e.g. [48]) and $\tilde{n}=2 L-n$. It is easy to prove the following properties of $f_{n, L}(x)$

$$
x^{n} f_{n, L}\left(\frac{1}{x}\right)=f_{n, L}(x), \quad x^{n-L} f_{2 L-n, L}(x)=f_{n, L}(x) .
$$

A straightforward application of these is the relation

$$
f_{2 L-n, L}(x)=x^{L} f_{n, L}\left(\frac{1}{x}\right)
$$

which can be used to get an upper bound

$$
\left(\begin{array}{c}
L \\
2 L-n
\end{array}\right){ }_{2} F_{1}[-L, n-2 L ; 1+n-L ; 2]<2^{L}\left(\begin{array}{c}
2 L \\
n
\end{array}\right) .
$$

$f_{2 L-n, L}(x)$ is a polynomial of degree $2 L-n$ and thus has $2 L-n$ roots. Because of Descartes rule and the properties of Jacobi polynomials all the zeros are real, distinct and negative. Furthermore because of (B.10)

$$
f_{2 L-n, L}(x)=c \prod_{j=1}^{L-n / 2}\left(x+\left|x_{j}\right|\right)\left(x+\frac{1}{\left|x_{j}\right|}\right)
$$

where all $x_{j} \in(-1,0)$. We thus can write ${ }^{6}$

$$
\left|\frac{f_{2 L-n, L}(2)}{f_{2 L-n, L}(-2)}\right|=\left|\frac{{ }_{2} F_{1}[-L, n-2 L ; 1+n-L ; 2]}{{ }_{2} F_{1}[-L, n-2 L, 1+n-L,-2]}\right|=\prod_{j=1}^{L-n / 2}\left|\frac{\left|x_{j}\right|+2}{\left|x_{j}\right|-2}\right|\left|\frac{1 /\left|x_{j}\right|+2}{1 /\left|x_{j}\right|-2}\right| .
$$

If the roots are densely distributed the right-hand side grows exponentially with growing $L$. To say something more about the root distribution we observe that

$$
f_{2 L-n, L}(x)=P_{\tilde{n}}^{(L-\tilde{n},-2 L-1)}(1-2 x)=(1-x)^{2 L-n} P_{2 L-n}^{n-L, n-L}\left(\frac{x+1}{1-x}\right) .
$$

Since $P_{m}^{(\alpha, \alpha)}(y)$ with $\alpha>-1$ has roots densely distributed on $(-1,1)$ we conclude that the roots of $f_{2 L-n, L}(x)$ are densely distributed everywhere on $(-\infty, 0)$ which completes the proof.

\section{Appendix C. Exact results for finite $L$}

Here we present the energy for the field strength operators (1.6) of finite length $L$ in powers of the coupling constant. Their anomalous dimension is (2.8). The results were obtained using Mathematica. The $\zeta$-functions are exclusively generated by the dressing factor.

\footnotetext{
${ }^{6}$ For simplicity assume $x_{j} \neq-1 / 2$.
} 
$\mathbf{L}=3:^{7}$

$E_{3}=9-\frac{81}{2} g^{2}+315 g^{4}+\cdots$

$\mathrm{L}=4$ :

$$
\begin{aligned}
E_{4}=12-50 g^{2} & +\frac{1515}{4} g^{4}-\left(\frac{513937}{144}+216 \zeta(3)\right) g^{6} \\
& +\left(\frac{22129823}{576}+3302 \zeta(3)+2160 \zeta(5)\right) g^{8}+\cdots
\end{aligned}
$$

$\mathrm{L}=5$ :

$$
\begin{aligned}
E_{5}=15-\frac{129}{2} & g^{2}+\frac{39411}{80} g^{4}-\left(\frac{7346253}{1600}+270 \zeta(3)\right) g^{6} \\
& +\left(\frac{1539949881}{32000}+\frac{8307 \zeta(3)}{2}+2700 \zeta(5)\right) g^{8}+\cdots
\end{aligned}
$$

$\mathrm{L}=6$ :

$$
\begin{aligned}
E_{6}=18-\frac{837}{11} & g^{2}+\frac{6278355}{10648} g^{4}-\left(\frac{29266837713}{5153632}+324 \zeta(3)\right) g^{6} \\
& +\left(\frac{76857234976107}{1247178944}+\frac{605205 \zeta(3)}{121}+3240 \zeta(5)\right) g^{8}+\cdots
\end{aligned}
$$

$\mathbf{L}=7$ :

$$
\begin{aligned}
E_{7}=21-\frac{179}{2} & g^{2}+\frac{76603}{112} g^{4}-\left(\frac{181131695}{28224}+378 \zeta(3)\right) g^{6} \\
& +\left(\frac{8959397257}{131712}+\frac{11641 \zeta(3)}{2}+3780 \zeta(5)\right) g^{8}+\cdots
\end{aligned}
$$

$\mathbf{L}=8$ :

$$
\begin{aligned}
E_{8}=24-\frac{4380}{43} & g^{2}+\frac{125533809}{159014} g^{4}-\left(\frac{8840715968859}{1176067544}+432 \zeta(3)\right) g^{6} \\
& +\left(\frac{346753221469919673}{4349097777712}+\frac{12323484 \zeta(3)}{1849}+4320 \zeta(5)\right) g^{8}+\cdots .
\end{aligned}
$$

There are several conclusions one can draw from these exact results as compared to (4.36). It is clear that the higher loop corrections are not exactly proportional to $L$ for finite length operators. This is presumably caused by length changing processes, see [8]. Also, the transcendentality principle is violated. The terms of highest transcendentality, however, are strictly proportional to $L$ and precisely agree with the ones derived in (4.36).

\section{Appendix D. Emulation of the dressing phase?}

In this paper we have argued that a nested Bethe ansatz naturally gives rise to a convolution structure very similar to the one in the proposed all-loop dressing phase.

${ }^{7}$ Please note that for this particular 'short' state wrapping effects compete with the deformation caused by the dressing factor. The Bethe equations are not reliable anymore, and we thus omit the $\mathcal{O}\left(g^{6}\right)$ term. 
In this appendix ${ }^{8}$ we will ask whether we can modify or supplement the asymptotic Bethe equations of [8] in order to construct a well-defined set of equations leading to the correct dressing phase.

We notice that the difference between the nesting phase (1.9) and the dressing phase (1.2) is just, apart from a factor of two, a minus sign in the diagonal kernel $1 /\left(\mathrm{e}^{t} \pm 1\right)$ inside the convolution. The factor of two is easily fixed by using two instead of one set of auxiliary roots, just as in the case of the $\mathfrak{s o}(6)$ nesting, see (5.12). We claim that the sign may also be formally fixed by emulating the full set of asymptotic Bethe equations with dressing factor by the following set of equations:

$$
\begin{aligned}
1=\left(\frac{x_{4, k}^{+}}{x_{4, k}^{-}}\right)^{2 M_{\mathrm{d}}-L} & \prod_{\substack{j=1 \\
j \neq k}}^{K_{4}}\left(\frac{x_{4, k}^{+}-x_{4, j}^{-}}{x_{4, k}^{-}-x_{4, j}^{+}} \frac{1-g^{2} / x_{4, k}^{+} x_{4, j}^{-}}{1-g^{2} / x_{4, k}^{-} x_{4, j}^{+}}\right) \\
& \times \prod_{j=1}^{K_{1}} \frac{1-g^{2} / x_{4, k}^{-} x_{1, j}}{1-g^{2} / x_{4, k}^{+} x_{1, j}} \prod_{j=1}^{K_{3}} \frac{x_{4, k}^{-}-x_{3, j}}{x_{4, k}^{+}-x_{3, j}} \prod_{j=1}^{K_{5}} \frac{x_{4, k}^{-}-x_{5, j}}{x_{4, k}^{+}-x_{5, j}} \prod_{j=1}^{K_{7}} \frac{1-g^{2} / x_{4, k}^{-} x_{7, j}}{1-g^{2} / x_{4, k}^{+} x_{7, j}} \\
& \times \prod_{j=1}^{M_{\mathrm{d}}} \frac{1-g^{2} / x_{4, k}^{-} x_{\overline{1}, j}}{1-g^{2} / x_{4, k}^{+} x_{\overline{1}, j}} \prod_{j=1}^{M_{\mathrm{d}}} \frac{x_{4, k}^{-}-x_{\overline{3}, j}}{x_{4, k}^{+}-x_{\overline{3}, j}} \prod_{j=1}^{x_{4, k}-x_{\overline{5}, j}} \frac{x_{\mathrm{d}}^{-}}{x_{4, k}^{+}-x_{\overline{5}, j}} \frac{1-g^{2} / x_{4, k}^{-} x_{\overline{7}, j}}{1-g^{2} / x_{4, k}^{+} x_{\overline{7}, j}},
\end{aligned}
$$

$1=\prod_{j=1}^{M_{\mathrm{d}}} \frac{u_{\overline{1}, k}-u_{\overline{2}, j}+\mathrm{i} / 2}{u_{\overline{1}, k}-u_{\overline{2}, j}-\mathrm{i} / 2} \prod_{j=1}^{K_{4}} \frac{1-g^{2} / x_{\overline{1}, k} x_{4, j}^{+}}{1-g^{2} / x_{\overline{1}, k} x_{4, j}^{-}}$

$1=\prod_{\substack{j=1 \\ j \neq k}}^{M_{\mathrm{d}}} \frac{u_{\overline{2}, k}-u_{\overline{2}, j}-\mathrm{i}}{u_{\overline{2}, k}-u_{\overline{2}, j}+\mathrm{i}} \prod_{j=1}^{M_{\mathrm{d}}} \frac{u_{\overline{2}, k}-u_{\overline{3}, j}+\mathrm{i} / 2}{u_{\overline{2}, k}-u_{\overline{3}, j}-\mathrm{i} / 2} \prod_{j=1}^{M_{\mathrm{d}}} \frac{u_{\overline{2}, k}-u_{\overline{1}, j}+\mathrm{i} / 2}{u_{\overline{2}, k}-u_{\overline{1}, j}-\mathrm{i} / 2}$,

$1=\prod_{j=1}^{M_{\mathrm{d}}} \frac{u_{\overline{3}, k}-u_{\overline{2}, j}+\mathrm{i} / 2}{u_{\overline{3}, k}-u_{\overline{2}, j}-\mathrm{i} / 2} \prod_{j=1}^{K_{4}} \frac{x_{\overline{3}, k}-x_{4, j}^{+}}{x_{\overline{3}, k}-x_{4, j}^{-}}$,

$1=\prod_{j=1}^{K_{2}} \frac{u_{1, k}-u_{2, j}+\mathrm{i} / 2}{u_{1, k}-u_{2, j}-\mathrm{i} / 2} \prod_{j=1}^{K_{4}} \frac{1-g^{2} / x_{1, k} x_{4, j}^{+}}{1-g^{2} / x_{1, k} x_{4, j}^{-}}$

$1=\prod_{\substack{j=1 \\ j \neq k}}^{K_{2}} \frac{u_{2, k}-u_{2, j}-\mathrm{i}}{u_{2, k}-u_{2, j}+\mathrm{i}} \prod_{j=1}^{K_{3}} \frac{u_{2, k}-u_{3, j}+\mathrm{i} / 2}{u_{2, k}-u_{3, j}-\mathrm{i} / 2} \prod_{j=1}^{K_{1}} \frac{u_{2, k}-u_{1, j}+\mathrm{i} / 2}{u_{2, k}-u_{1, j}-\mathrm{i} / 2}$,

$1=\prod_{j=1}^{K_{2}} \frac{u_{3, k}-u_{2, j}+\mathrm{i} / 2}{u_{3, k}-u_{2, j}-\mathrm{i} / 2} \prod_{j=1}^{K_{4}} \frac{x_{3, k}-x_{4, j}^{+}}{x_{3, k}-x_{4, j}^{-}}$,

\footnotetext{
8 The results of appendix D were known to us for some time, but we did not include them in an earlier version of this paper due to the various difficulties discussed below. While preparing an improved version of this paper similar ideas appeared in [37]. We therefore decided to include our notes in order to discuss the problems which would have to be resolved in order to make this and similar mechanisms viable. Furthermore, our procedure differs in several ways from [37]. In particular, we distinguish nesting from dressing roots (there is no direct interaction between them), and we do not assume a macroscopic number of momentum-carrying main roots.
} 


$$
\begin{aligned}
& 1=\prod_{j=1}^{K_{6}} \frac{u_{5, k}-u_{6, j}+\mathrm{i} / 2}{u_{5, k}-u_{6, j}-\mathrm{i} / 2} \prod_{j=1}^{K_{4}} \frac{x_{5, k}-x_{4, j}^{+}}{x_{5, k}-x_{4, j}^{-}}, \\
& 1=\prod_{\substack{j=1 \\
j \neq k}}^{K_{6}} \frac{u_{6, k}-u_{6, j}-\mathrm{i}}{u_{6, k}-u_{6, j}+\mathrm{i}} \prod_{j=1}^{K_{5}} \frac{u_{6, k}-u_{5, j}+\mathrm{i} / 2}{u_{6, k}-u_{5, j}-\mathrm{i} / 2} \prod_{j=1}^{K_{7}} \frac{u_{6, k}-u_{7, j}+\mathrm{i} / 2}{u_{6, k}-u_{7, j}-\mathrm{i} / 2}, \\
& 1=\prod_{j=1}^{K_{6}} \frac{u_{7, k}-u_{6, j}+\mathrm{i} / 2}{u_{7, k}-u_{6, j}-\mathrm{i} / 2} \prod_{j=1}^{K_{4}} \frac{1-g^{2} / x_{7, k} x_{4, j}^{+}}{1-g^{2} / x_{7, k} x_{4, j}^{-}},
\end{aligned}
$$

$$
\begin{aligned}
1 & =\prod_{j=1}^{M_{\mathrm{d}}} \frac{u_{\overline{5}, k}-u_{\overline{6}, j}+\mathrm{i} / 2}{u_{\overline{5}, k}-u_{\overline{6}, j}-\mathrm{i} / 2} \prod_{j=1}^{K_{4}} \frac{x_{\overline{5}, k}-x_{4, j}^{+}}{x_{\overline{5}, k}-x_{4, j}^{-}}, \\
1 & =\prod_{\substack{j=1 \\
j \neq k}}^{M_{\mathrm{d}}} \frac{u_{\overline{6}, k}-u_{\overline{6}, j}-\mathrm{i}}{u_{\overline{6}, k}-u_{\overline{6}, j}+\mathrm{i}} \prod_{j=1}^{M_{\mathrm{d}}} \frac{u_{\overline{6}, k}-u_{\overline{5}, j}+\mathrm{i} / 2}{u_{\overline{6}, k}-u_{\overline{5}, j}-\mathrm{i} / 2} \prod_{j=1}^{M_{\mathrm{d}}} \frac{u_{\overline{6}, k}-u_{\overline{7}, j}+\mathrm{i} / 2}{u_{\overline{6}, k}-u_{\overline{7}, j}-\mathrm{i} / 2}, \\
1 & =\prod_{j=1}^{M_{\mathrm{d}}} \frac{u_{\overline{7}, k}-u_{\overline{6}, j}+\mathrm{i} / 2}{u_{\overline{7}, k}-u_{\overline{6}, j}-\mathrm{i} / 2} \prod_{j=1}^{K_{4}} \frac{1-g^{2} / x_{\overline{7}, k} x_{4, j}^{+}}{1-g^{2} / x_{\overline{7}, k} x_{4, j}^{-}} .
\end{aligned}
$$

These equations are very similar to the ones in table 5 of [8]. However, the dressing factor $\prod \sigma^{2}$ of table 5 is now missing and is supposed to be emulated by

$\sigma_{k}^{2}\left(x_{4,1}, \ldots, x_{4, K_{4}}\right)=\prod_{j=1}^{M_{\mathrm{d}}} \frac{x_{4, k}^{-}-g^{2} / x_{\overline{1}, j}}{x_{4, k}^{+}-g^{2} / x_{\overline{1}, j}} \frac{x_{4, k}^{-}-x_{\overline{3}, j}}{x_{4, k}^{+}-x_{\overline{3}, j}} \frac{x_{4, k}^{-}-x_{\overline{5}, j}}{x_{4, k}^{+}-x_{\overline{5}, j}} \frac{x_{4, k}^{-}-g^{2} / x_{\overline{7}, j}}{x_{4, k}^{+}-g^{2} / x_{\overline{7}, j}}$.

Note that we have introduced an additional set of $2 \times 3 \times M_{\mathrm{d}}$ auxiliary 'dressing' roots denoted by a bar on the flavour indices $\{\overline{1}, \overline{2}, \overline{3}\},\{\overline{5}, \overline{6}, \overline{7}\}$. They should not be confused with the set of $3+1+3$ 'nesting' Bethe roots, carrying unbarred indices, with multiplicities $\left(K_{1}, K_{2}, K_{3}, K_{4}, K_{5}, K_{6}, K_{7}\right)$. These are identical to the ones in [8]. We will now prove our claim, which however requires the $M_{\mathrm{d}} \rightarrow \infty$ limit. We will also show, as required by consistency, that the emulated dressing phase indeed factorizes

$$
\sigma_{k}^{2}\left(x_{4,1}, \ldots, x_{4, K_{4}}\right)=\prod_{\substack{j=1 \\ j \neq k}}^{K_{4}} \sigma^{2}\left(x_{4, k}, x_{4, j}\right)
$$

as required by the asymptotic Bethe equations of table 5 in [8]. Notice that there are various ways to rewrite the equations (D.1)-(D.5) since we may always perform dynamic transformations [8] and therewith trade back and forth, respectively, roots of type $\overline{1}, \overline{7}$ and type $\overline{3}, \overline{5}$.

The derivation of the dressing factor formally proceeds as in the main body of this article. We will assume the same mechanism of stack formation. We may then eliminate the dressing roots of type $\{\overline{1}, \overline{3}\}$ and $\{\overline{5}, \overline{7}\}$ and write 'effective' equations coupling the 
dressing roots of type $\overline{2}$ and $\overline{6}$ to the momentum-carrying roots of type 4 . This yields

$$
\begin{gathered}
1=\prod_{\substack{j=1 \\
j \neq k}}^{M_{\mathrm{d}}} \frac{u_{\overline{2}, k}-u_{\overline{2}, j}-\mathrm{i}}{u_{\overline{2}, k}-u_{\overline{2}, j}+\mathrm{i}} \prod_{j=1}^{K_{4}} \frac{1-g^{2} / x_{\overline{2}, k}^{+} x_{4, j}^{-}}{1-g^{2} / x_{\overline{2}, k}^{-} x_{4, j}^{+}} \frac{1-g^{2} / x_{\overline{2}, k}^{-} x_{4, j}^{-}}{1-g^{2} / x_{\overline{2}, k}^{+} x_{4, j}^{+}}, \\
\sigma_{k}^{2}\left(x_{4,1}, \ldots, x_{4, K_{4}}\right) \\
=\prod_{j=1}^{M_{\mathrm{d}}} \frac{1-g^{2} / x_{4, k}^{-} x_{\overline{2}, j}^{+}}{1-g^{2} / x_{4, k}^{+} x_{\overline{2}, j}^{-}} \frac{1-g^{2} / x_{4, k}^{-} x_{\overline{2}, j}^{-}}{1-g^{2} / x_{4, k}^{+} x_{\overline{2}, j}^{+}} \\
\quad \times \prod_{j=1}^{M_{\mathrm{d}}} \frac{1-g^{2} / x_{4, k}^{-} x_{\overline{6}, j}^{+}}{1-g^{2} / x_{4, k}^{+} x_{\overline{6}, j}^{-}} \frac{1-g^{2} / x_{4, k}^{-} x_{\overline{6}, j}^{-}}{1-g^{2} / x_{4, k}^{+} x_{\overline{6}, j}^{+}} \\
1=\prod_{\substack{j=1 \\
j \neq k}}^{M_{\mathrm{d}}} \frac{u_{\overline{6}, k}-u_{\overline{6}, j}-\mathrm{i}}{u_{\overline{6}, k}-u_{\overline{6}, j}+\mathrm{i}} \prod_{j=1}^{K_{4}} \frac{1-g^{2} / x_{\overline{6}, k}^{+} x_{4, j}^{-}}{1-g^{2} / x_{\overline{6}, k}^{-} x_{4, j}^{+}} \frac{1-g^{2} / x_{\overline{6}, k}^{-} x_{4, j}^{-}}{1-x_{\overline{6}, k}^{+} x_{4, j}^{+}} .
\end{gathered}
$$

We should however mention that these equations do not have any proper solutions for finite values of $M_{\mathrm{d}}$. Nevertheless, one may formally proceed and take $M_{\mathrm{d}}$ to infinity. Note that we do not assume any of the original quantum numbers $\left(L ; K_{1}, K_{2}, K_{3}, K_{4}, K_{5}, K_{6}, K_{7}\right)$ of the asymptotic ansatz to be thermodynamically large. The remainder of the derivation proceeds again by transforming (D.10) into Fourier space

$0=\mathrm{e}^{|t|} \hat{\xi}(t)-\hat{\xi}(t)-\frac{1}{2 \pi M_{\mathrm{d}}} \int_{-\infty}^{\infty} \mathrm{d} t^{\prime} \sum_{j=1}^{K_{4}} \mathrm{e}^{-\mathrm{i} t^{\prime} u_{j}} \mathrm{e}^{|t|}\left(\hat{K}\left(2 g t, 2 g t^{\prime}\right)+\hat{H}\left(2 g t, 2 g t^{\prime}\right)\right) \mathrm{e}^{\left|t^{\prime}\right| / 2}$

where $\hat{K}\left(t, t^{\prime}\right)$ and $\hat{H}\left(t, t^{\prime}\right)$ are given by (4.27) and (4.28) and $\hat{\xi}(t)$ is the density of the dressing roots. If we do not assume the distribution of roots to be an even function, we end up with two dressing densities, for $t>0$ and $t<0$ respectively

$\hat{\xi}( \pm t)=\frac{-2 g^{2} t}{M_{\mathrm{d}}} \frac{1}{\mathrm{e}^{t}-1} \int_{0}^{\infty} \mathrm{d} t^{\prime} \mathrm{e}^{-t^{\prime} / 2}\left(\sum_{j=1}^{K_{4}} \mathrm{e}^{ \pm \mathrm{i}^{\prime} u_{j}} \hat{K}_{m}\left(2 g t, 2 g t^{\prime}\right)+\sum_{j=1}^{K_{4}} \mathrm{e}^{\mp \mathrm{i} t^{\prime} u_{j}} \hat{H}_{m}\left(2 g t, 2 g t^{\prime}\right)\right)$

with $\hat{K}_{m}$ given by (4.33) and

$$
\hat{H}_{m}\left(t, t^{\prime}\right)=\frac{J_{0}(t) J_{1}\left(t^{\prime}\right)+J_{0}\left(t^{\prime}\right) J_{1}(t)}{t+t^{\prime}} .
$$

Due to symmetry the second set of dressing roots can be treated in the same way. After elimination of the auxiliary densities in (D.9) one finds

$$
\begin{aligned}
2 \mathrm{i} \theta_{k}\left(x_{1}, \ldots, x_{M}\right) & =\arg \sigma_{k}^{2}\left(x_{1}, \ldots, x_{M}\right) \\
= & 2 g^{2} \int_{-\infty}^{\infty} \mathrm{d} t \mathrm{e}^{-|t| / 2} \mathrm{e}^{\mathrm{i} t u_{k}} \int_{-\infty}^{\infty} \mathrm{d} t^{\prime} \mathrm{e}^{-\left|t^{\prime}\right| / 2} \sum_{j=1}^{M} \mathrm{e}^{\mathrm{i} t^{\prime} u_{j}}\left(\hat{K}_{\mathrm{d}}\left(2 g t^{\prime}, 2 g t\right)-\hat{K}_{\mathrm{d}}\left(2 g t, 2 g t^{\prime}\right)\right)
\end{aligned}
$$

with the dressing kernel given by (1.2). Comparing the last equation with (1.1) we notice that the dressing phase factorizes as promised in (D.7). 


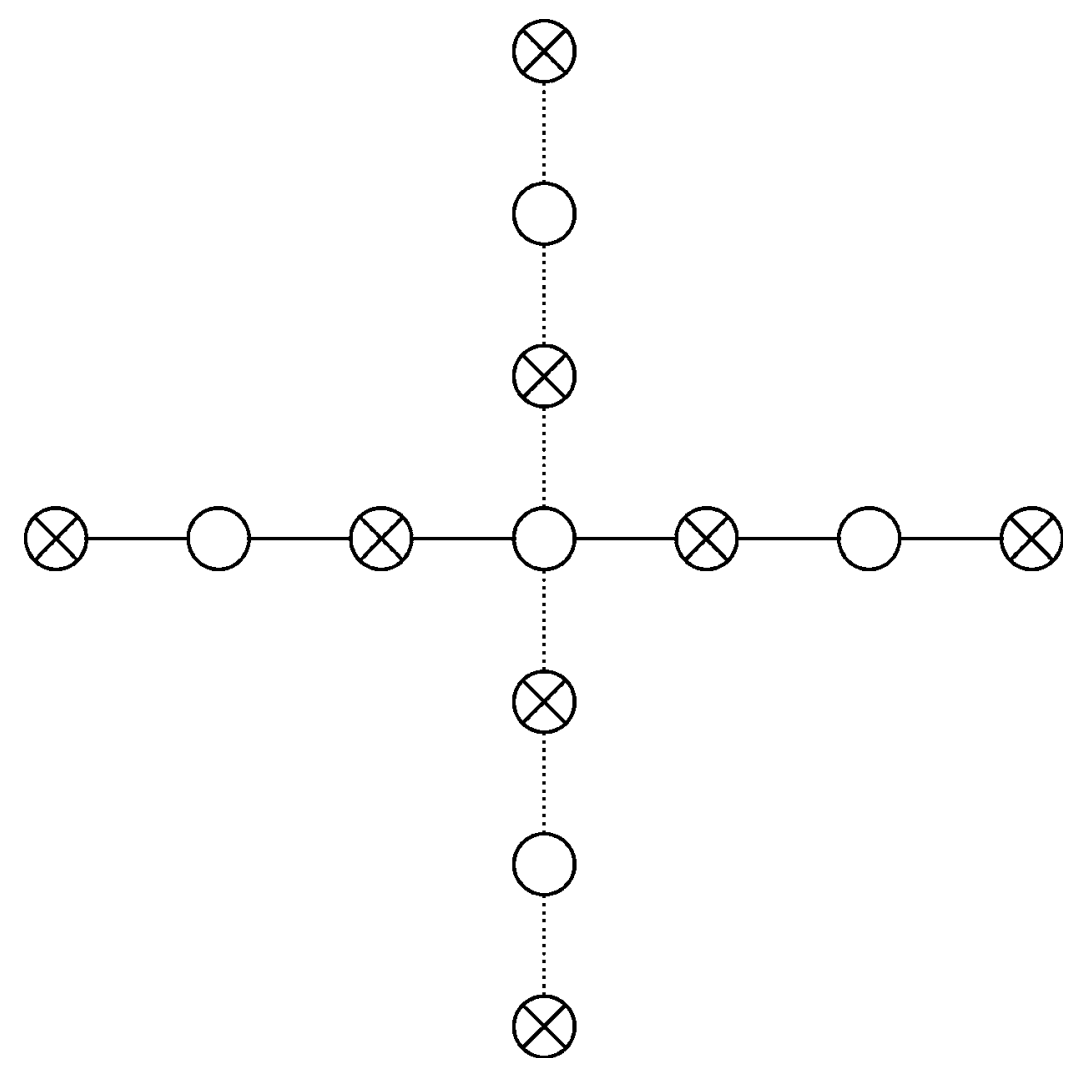

Figure D.1. Schematic scattering notation for the dressed asymptotic Bethe ansatz equations (D.1)-(D.5). The horizontal branch represents the Dynkin diagram of $\mathfrak{p s u}(2,2 \mid 4)$ and the scattering of elementary magnons. The vertical branch (dotted lines) indicates the emulation of the dressing phase by the scattering of the dressing roots among themselves and the momentum-carrying main node. Note that the auxiliary 'dressing' roots do not scatter with any of the auxiliary 'nesting' roots.

The above procedure is suggestive, but currently plagued by the following problems:

- There is no solution to the equations (D.2) and (D.5) relating to the dressing roots when their number $M_{\mathrm{d}}$ is finite. Therefore expressions such as (D.11), where we employ $M_{\mathrm{d}}$ as an infrared regulator, are somewhat ill-defined. This is in contradistinction to our treatment of $\mathcal{F}^{L}$ where the equations certainly make sense at finite $L$.

- The scattering pattern of (D.1)-(D.5) corresponds to a very curious 'Dynkin diagram' whose Lie-algebraic origin is very questionable, see figure D.1. Furthermore, the occupation (filling) numbers appear to be inconsistent with a standard nested Bethe ansatz. It would have to be shown how such filling numbers can be obtained from the non-compact nature of the underlying system.

- The ansatz (D.1)-(D.5) leads to Bethe equations which are at $M_{\mathrm{d}}=\infty$ strictly identical to the original ones with the dressing factor. So the problem of the asymptotic character of the equations of [8] is not improved, and finite size effects are still not properly implemented. 
- Finally, it is completely unclear which 'hidden' excitations are supposed to be scattering according to (D.1)-(D.5). It is certainly not clear what, if anything, is being diagonalized by these equations. The equations might however turn out to be useful for proving the crossing invariance of the dressing factor at finite values of the coupling constant.

\section{References}

[1] Minahan J A and Zarembo K, The Bethe-ansatz for $\mathcal{N}=4$ super Yang-Mills, 2003 J. High Energy Phys. JHEP03(2003)013 [hep-th/0212208]

[2] Beisert N and Staudacher M, The $\mathcal{N}=4$ SYM integrable super spin chain, 2003 Nucl. Phys. B $\mathbf{6 7 0} 439$ [hep-th/0307042]

[3] Lipatov L N, Evolution equations in QCD, 1998 Proc. Conf. on Perspectives in Hadronic Physics (ICTP, Trieste, May 1997) ed S Boffi, C Ciofi Degli Atti and M Giannini (Singapore: World Scientific)

[4] Braun V M, Derkachov S E and Manashov A N, Integrability of three-particle evolution equations in QCD, 1998 Phys. Rev. Lett. 812020 [hep-ph/9805225]

Braun V M, Derkachov S E, Korchemsky G P and Manashov A N, Baryon distribution amplitudes in $Q C D$, 1999 Nucl. Phys. B 553355 [hep-ph/9902375]

Belitsky A V, Fine structure of spectrum of twist-three operators in QCD, 1999 Phys. Lett. B 45359 [hep-ph/9902361]

Belitsky A V, Renormalization of twist-three operators and integrable lattice models, 2000 Nucl. Phys. B $\mathbf{5 7 4} 407$ [hep-ph/9907420]

[5] Staudacher M, The factorized S-matrix of CFT/AdS, 2005 J. High Energy Phys. JHEP05(2005)054 [hep-th/0412188]

[6] Arutyunov G, Frolov S and Staudacher M, Bethe ansatz for quantum strings, 2004 J. High Energy Phys. JHEP10(2004)016 [hep-th/0406256]

[7] Beisert N, Kristjansen $\mathrm{C}$ and Staudacher M, The dilatation operator of $\mathcal{N}=4$ super Yang-Mills theory, 2003 Nucl. Phys. B 664131 [hep-th/0303060]

Beisert N, The $\mathfrak{s u}(2 \mid 3)$ dynamic spin chain, 2004 Nucl. Phys. B 682487 [hep-th/0310252]

Serban D and Staudacher M, Planar $\mathcal{N}=4$ gauge theory and the Inozemtsev long range spin chain, $2004 \mathrm{~J}$. High Energy Phys. JHEP06(2004)001 [hep-th/0401057]

Beisert N, Dippel V and Staudacher M, A novel long range spin chain and planar $\mathcal{N}=4$ super Yang-Mills, 2004 J. High Energy Phys. JHEP07(2004)075 [hep-th/0405001]

[8] Beisert N and Staudacher M, Long-range $\mathfrak{p s u}(2,2 \mid 4)$ Bethe ansätze for gauge theory and strings, 2005 Nucl. Phys. B 7271 [hep-th/0504190]

[9] Beisert N, The $\mathfrak{s u}(2 \mid 2)$ dynamic S-matrix, 2005 Preprint hep-th/0511082

Beisert N, The analytic Bethe ansatz for a chain with centrally extended $\mathfrak{s u}(2 \mid 2)$ symmetry, 2007 J. Stat. Mech. P01017 [nlin.SI/0610017]

[10] Arutyunov G, Frolov S and Zamaklar M, The Zamolodchikov-Faddeev algebra for AdS $S_{5} \times S^{5}$ superstring, 2006 Preprint hep-th/0612229

[11] Janik R A, The AdS $S_{5} \times S^{5}$ superstring worldsheet $S$-matrix and crossing symmetry, 2006 Phys. Rev. D 73086006 [hep-th/0603038]

[12] Hernandez R and Lopez E, Quantum corrections to the string Bethe ansatz, 2006 J. High Energy Phys. JHEP07(2006)004 [hep-th/0603204]

[13] Arutyunov G and Frolov S, On AdS $S_{5} \times S^{5}$ string S-matrix, 2006 Phys. Lett. B 639378 [hep-th/0604043]

[14] Beisert N, Eden B and Staudacher M, Transcendentality and crossing, 2007 J. Stat. Mech. P01021 [hep-th/0610251]

[15] Eden B and Staudacher M, Integrability and transcendentality, 2006 J. Stat. Mech. P11014 [hep-th/0603157]

[16] Beisert N, Hernández R and López E, A crossing-symmetric phase for AdS $S_{5} \times S^{5}$ strings, 2006 J. High Energy Phys. JHEP11(2006)070 [hep-th/0609044]

[17] Bern Z, Czakon M, Dixon L J, Kosower D A and Smirnov V A, The four-loop planar amplitude and cusp anomalous dimension in maximally supersymmetric Yang-Mills theory, 2006 Preprint hep-th/0610248

[18] Cachazo F, Spradlin M and Volovich A, Four-loop cusp anomalous dimension from obstructions, 2006 Preprint hep-th/0612309 
[19] Kotikov A V and Lipatov L N, On the highest transcendentality in $\mathcal{N}=4$ SUSY, 2006 Preprint hep-th/0611204

[20] Gubser S S, Klebanov I R and Polyakov A M, A semi-classical limit of the gauge/string correspondence, 2002 Nucl. Phys. B 63699 [hep-th/0204051]

[21] Benna M K, Benvenuti S, Klebanov I R and Scardicchio A, A test of the AdS/CFT correspondence using high-spin operators, 2006 Preprint hep-th/0611135

[22] Alday L F, Arutyunov G, Benna M K, Eden B and Klebanov I R, On the strong coupling scaling dimension of high spin operators, 2007 Preprint hep-th/0702028

[23] Kostov I, Serban D and Volin D, Strong coupling limit of Bethe ansatz equations, 2007 Preprint hep-th/0703031

[24] Beccaria M, De Angelis G F and Forini V, The scaling function at strong coupling from the quantum string Bethe equations, 2007 Preprint hep-th/0703131

[25] Frolov S and Tseytlin A A, Semiclassical quantization of rotating superstring in $A d S_{5} \times S^{5}, 2002 \mathrm{~J}$. High Energy Phys. JHEP06(2002)007 [hep-th/0204226]

[26] Rej A, Serban D and Staudacher M, Planar $\mathcal{N}=4$ gauge theory and the Hubbard model, 2006 J. High Energy Phys. JHEP03(2006)018 [hep-th/0512077]

[27] Mann N and Polchinski J, Finite density states in integrable conformal field theories, 2005 From Fields to Strings: Circumnavigating Theoretical Physics, in Memory of Ian Kogan (From Fields to Strings vol 2) ed M Shifman, A Vainshtein and J Wheater (Singapore: World Scientific) pp 1365-83 [hep-th/0408162]

Mann N and Polchinski J, Bethe ansatz for a quantum supercoset sigma model, 2005 Phys. Rev. D 72086002 [hep-th/0508232]

[28] Gromov N and Kazakov V, Asymptotic Bethe ansatz from string sigma model on $S^{3} \times R, 2006$ Preprint hep-th/0605026

[29] Beisert N, The dilatation operator of $N=4$ super Yang-Mills theory and integrability, 2005 Phys. Rep. 4051 [hep-th/0407277]

[30] Ferretti G, Heise R and Zarembo K, New integrable structures in large-N QCD, 2004 Phys. Rev. D 70074024 [hep-th/0404187]

Beisert N, Ferretti G, Heise R and Zarembo K, One-loop QCD spin chain and its spectrum, 2005 Nucl. Phys. B 717137 [hep-th/0412029]

[31] Arutyunov G and Tseytlin A A, On highest-energy state in the $\mathfrak{s u}(1 \mid 1)$ sector of $\mathcal{N}=4$ super Yang-Mills theory, 2006 J. High Energy Phys. JHEP05(2006)033 [hep-th/0603113]

[32] Beisert N, Kazakov V A, Sakai K and Zarembo K, Complete spectrum of long operators in $\mathcal{N}=4 S Y M$ at one loop, 2005 J. High Energy Phys. JHEP07(2005)030 [hep-th/0503200]

[33] Serban D and Staudacher M, 2005 unpublished

[34] Zarembo K, Antiferromagnetic operators in $\mathcal{N}=4$ supersymmetric Yang-Mills theory, 2006 Phys. Lett. B $\mathbf{6 3 4} 552$ [hep-th/0512079]

[35] Reshetikhin N Y, $O(N)$ invariant quantum field theoretical models: exact solution, 1985 Nucl. Phys. B $\mathbf{2 5 1 5 6 5}$

[36] Beccaria M and Ortix C, AdS/CFT duality at strong coupling, 2006 Preprint hep-th/0610215

Beccaria M, De Angelis G F, Del Debbio L and Picariello M, Highest states in light-cone $A d S_{5} \times S^{5}$ superstring, 2007 Preprint hep-th/0701167

[37] Sakai K and Satoh Y, Origin of dressing phase in N=4 super Yang-Mills, 2007 Preprint hep-th/0703177

[38] Ambjorn J, Janik R A and Kristjansen C, Wrapping interactions and a new source of corrections to the spin-chain/string duality, 2006 Nucl. Phys. B 736288 [hep-th/0510171]

[39] Schafer-Nameki S, Zamaklar M and Zarembo K, How accurate is the quantum string Bethe ansatz?, $2006 \mathrm{~J}$. High Energy Phys. JHEP12(2006)020 [hep-th/0610250]

[40] Hofman D M and Maldacena J M, Giant magnons, 2006 J. Phys. A: Math. Gen. 3913095 [hep-th/0604135]

[41] Arutyunov G, Frolov S and Zamaklar M, Finite-size effects from giant magnons, 2006 Preprint hep-th/0606126

[42] Astolfi D, Forini V, Grignani G and Semenoff G W, Gauge invariant finite size spectrum of the giant magnon, 2007 Preprint hep-th/0702043

[43] Okamura K and Suzuki R, A perspective on classical strings from complex sine-Gordon solitons, 2007 Phys. Rev. D 75046001 [hep-th/0609026]

[44] Feverati G, Fioravanti D, Grinza P and Rossi M, On the finite size corrections of anti-ferromagnetic anomalous dimensions in $N=4$ SYM, 2006 J. High Energy Phys. JHEP05(2006)068 [hep-th/0602189]

Feverati G, Fioravanti D, Grinza P and Rossi M, Hubbard's adventures in $N=4$ SYM-land? Some non-perturbative considerations on finite length operators, 2006 Preprint hep-th/0611186 
[45] Klose T and Zarembo K, Bethe ansatz in stringy sigma models, 2006 J. Stat. Mech. P05006 [hep-th/0603039]

[46] Bytsko A G and Teschner J, Quantization of models with non-compact quantum group symmetry: modular XXZ magnet and lattice sinh-Gordon model, 2006 J. Phys. A: Math. Gen. 3912927 [hep-th/0602093]

[47] Belitsky A V, Derkachov S E, Korchemsky G P and Manashov A N, Baxter Q-operator for graded $\mathfrak{s l}(2 \mid 1)$ spin chain, 2007 J. Stat. Mech. P01005 [hep-th/0610332]

[48] Szegö G, Orthogonal polynomials, 1991 Colloquium Publication vol 23 (Providence, RI: American Mathematical Society) 